\title{
A STABILIZED FINITE VOLUME ELEMENT FORMULATION FOR SEDIMENTATION-CONSOLIDATION PROCESSES*
}

\author{
RAIMUND BÜRGER ${ }^{\dagger}$, RICARDO RUIZ-BAIER ${ }^{\ddagger}$, AND HÉCTOR TORRES§
}

\begin{abstract}
A model of sedimentation-consolidation processes in so-called clarifier-thickener units is given by a parabolic equation describing the evolution of the local solids concentration coupled with a version of the Stokes system for an incompressible fluid describing the motion of the mixture. In cylindrical coordinates, and if an axially symmetric solution is assumed, the original problem reduces to two space dimensions. This poses the difficulty that the subspaces for the construction of a numerical scheme involve weighted Sobolev spaces. A novel finite volume element method is introduced for the spatial discretization, where the velocity field and the solids concentration are discretized on two different dual meshes. The method is based on a stabilized discontinuous Galerkin formulation for the concentration field, and a multiscale stabilized pair of $\mathbb{P}_{1}-\mathbb{P}_{1}$ elements for velocity and pressure, respectively. Numerical experiments illustrate properties of the model and the satisfactory performance of the proposed method.
\end{abstract}

Key words. finite volume element method, axisymmetric formulation, sedimentation process, clarifier-thickener units

AMS subject classifications. 65M60, 65M08, 35Q35, 76D07

DOI. $10.1137 / 110836559$

\section{Introduction.}

1.1. Scope. The sedimentation of a suspension of small solid particles dispersed in a viscous fluid under the influence of gravity is a well-studied phenomenon and a fundamental unit operation in industrial applications and wastewater treatment. In many situations, the solid particles are flocculated artificially or naturally and form sediment layers that undergo consolidation. The modeling and simulation of the resulting sedimentation-consolidation process, and density-driven flows in general, requires the solution of a transport-flow problem in which a convection (or convectiondiffusion) equation for the transport of solids volume fraction is coupled with a version of the Stokes, Navier-Stokes, or Darcy equations describing the flow of the mixture. Solving these problems numerically both accurately and efficiently is difficult due to the combination of strong nonlinearities with the strong coupling between the field equations and the incompressibility constraint.

*Submitted to the journal's Computational Methods in Science and Engineering section June 6, 2011; accepted for publication (in revised form) February 17, 2012; published electronically May 29, 2012.

http://www.siam.org/journals/sisc/34-3/83655.html

${ }^{\dagger} \mathrm{CI}^{2} \mathrm{MA}$ and Departamento de Ingeniería Matemática, Universidad de Concepción, Casilla 160-C, Concepción, Chile (rburger@ing-mat.udec.cl). This author's work was supported by Conicyt (Chile) through Fondecyt project 1090456, BASAL project CMM, Universidad de Chile, and Centro de Investigación en Ingeniería Matemática $\left(\mathrm{CI}^{2} \mathrm{MA}\right)$.

¥Corresponding author. CMCS-MATHICSE-SB, École Polytechnique Fédérale de Lausanne, CH1015 Lausanne, Switzerland (ricardo.ruiz@epfl.ch). This author's work was supported by the European Research Council through the advanced grant "Mathcard, Mathematical Modelling and Simulation of the Cardiovascular System," ERC-2008-AdG 227058, and by CI ${ }^{2}$ MA, supported by Fondecyt project 1090456.

$\S$ Departamento de Matemáticas, Facultad de Ciencias, Universidad de La Serena, Av. Cisternas 1200, La Serena, Chile (htorres@userena.cl). This author's work was supported by Mecesup project UCO0713 and Fondecyt project 11110264. 
The physical principles of sedimentation-consolidation processes, corresponding mathematical models, and some numerical tools for their simulation in one or two space dimensions under simplifying assumptions can be found, e.g., in $[9,12]$ (see also $[14,53,54])$. The governing partial differential equations can be written as follows:

$$
\begin{aligned}
\partial_{t} \phi+\nabla \cdot \boldsymbol{F}(\phi, \boldsymbol{u}) & =\Delta A(\phi), \\
-\nabla \cdot(\mu(\phi) \boldsymbol{\varepsilon}(\boldsymbol{u})-\lambda p \mathbf{I}) & =\boldsymbol{G}(\phi), \\
\lambda \nabla \cdot \boldsymbol{u} & =0 \quad \text { in } \tilde{\Omega}, \quad t>0 .
\end{aligned}
$$

Here $\tilde{\Omega} \subset \mathbb{R}^{3}$ is a given spatial domain, and $t$ is time. The sought quantities are the local solids concentration $\phi$, the local volume-average velocity of the mixture $\boldsymbol{u}$, and the pressure $p$. Furthermore, $\boldsymbol{F}$ is a flux vector which is linear in $\boldsymbol{u}$ but nonlinear in $\phi$, $A$ is a nonlinear, nondecreasing diffusion function modeling sediment compressibility, $\mu(\phi) \boldsymbol{\varepsilon}(\boldsymbol{u})-\lambda p \mathbf{I}$ is the Cauchy stress tensor, where $\boldsymbol{\varepsilon}(\boldsymbol{u}):=\frac{1}{2}\left(\nabla \boldsymbol{u}+\nabla \boldsymbol{u}^{\mathrm{T}}\right), \lambda$ is a given coefficient, $\mu$ is a concentration-dependent viscosity function, and $\boldsymbol{G}$ is a forcing term modeling that local density fluctuations drive the motion of the mixture. The coupling of the Stokes system (1.1b), (1.1c) with the convection-diffusion equation (1.1a) induces several problems. First, at the discrete level, the convection-dominated nature of the problem results in failure of standard Galerkin approximations. Second, the method for the coupled problem also must include inf-sup stable discretizations for the Stokes system and at the same time guarantee (at least locally) conservation of mass. From a general mathematical perspective, the analysis of (1.1) faces other difficulties, including degeneracy where $A$ is flat, the possible need to introduce entropy conditions, and strong assumptions on the boundedness of the coupling terms.

The system (1.1) will be considered in an axisymmetric domain in two space dimensions along with initial and boundary conditions. This configuration represents widely used equipment in applications such as clarifier-thickeners in mineral processing or secondary settling tanks in wastewater treatment and avoids an excessive number of degrees of freedom, as would be required for a fully three-dimensional flow problem.

This paper develops, implements, and tests a numerical scheme for (1.1). In our proposed approach, the resulting system is discretized in time by a semi-implicit backward Euler method and in space by a suitable finite volume element (FVE) method whose main novelty lies in the formulation of a unified scheme for a coupled problem, while several FVE methods have been proposed for Stokes and quasi-linear scalar elliptic problems only; see, e.g., [7, 15, 48]. Besides the finite element (FE) primal mesh, we introduce two additional meshes on which we discretize velocity and solids fraction by continuous and discontinuous piecewise linear elements, respectively. Moreover, since the problem is defined in an axisymmetric setting, we need to employ weighted Sobolev spaces at both continuous and discrete levels.

1.2. Related work. An important amount of literature is devoted to the determination of exact and numerical solutions of model (1.1) in the one-dimensional case $[5,10,12,26,27,28,29,55]$ (this list is far from being complete). In that setting, only the equation for the concentration (1.1a) needs to be solved, while the mixture flow velocity is determined by boundary conditions. Only a limited number of papers, including $[11,24,30,37,40,41,49]$, deal with the numerical study of sedimentation-consolidation models in two or three space dimensions.

Fast and reliable numerical methods for related problems in two and three space dimensions include stable FE methods [37, 49], discontinuous Galerkin methods [18], finite volume (FV) formulations [5, 11], finite difference schemes [43, 47], and hybrid/combined FV-FE methods [16, 20, 33, 46]. The particular concept of FVE meth- 
ods (see [15]) is intermediate between finite volumes and finite elements. The ability of the numerical scheme to be locally conservative (as that of classical FV methods) while allowing for the deduction of $L^{2}$ estimates in a rather natural way (as for classical finite elements) is one of the most appealing features of FVE methods. In fact, the principle behind FVE methods is as follows. A Petrov-Galerkin type of discretization of the underlying problem is obtained, in which the test and trial functional spaces associated to the finite-dimensional problem do not originally coincide. With the aid of a transfer map, the test space is transformed to match the trial space. In this approach we further propose the use of two different transfer maps, which will allow us to project discrete functions defined in standard FE spaces associated to nodes and elements in a given primal mesh into different spaces associated to a dual and a diamond mesh. Using some properties of these transfer operators, the resulting FVE scheme adopts a form similar to the original Petrov-Galerkin formulation, which in our case corresponds to an edge-based stabilized method. The solution of the coupled transport-flow problem is then obtained by this final scheme.

In consonance with FE formulations for Navier-Stokes problems, to avoid spurious oscillations in the pressure approximation, inf-sup stable spaces are needed for the velocity-pressure pair. Among the possible discretizations of these variables, we restrict ourselves to continuous piecewise bilinear elements enriched with local functions for velocity $(\boldsymbol{u})$ and continuous piecewise linear elements for the pressure $(p)$ field (as in the multiscale stabilized method of $[2,48]$ ), whereas discontinuous piecewise linear elements are chosen for the approximation of the concentration $(\phi)$ field. In a diffusion-dominated regime, classical piecewise linear finite elements represent the most appropriate choice for the approximation of $\phi$. However, for convectiondominated problems, particular care must be taken to avoid nonphysical oscillations in $\phi$, which are likely to occur since solutions of degenerate parabolic equations, which include first-order hyperbolic conservation laws, exhibit sharp fronts and even shock discontinuities, which need to be resolved accurately. Such behavior motivates the use of discontinuous Galerkin elements for $\phi$. In that approach, no continuity across the interelement boundaries is required for the approximation functions. However, a connection between neighboring elements is enforced via a penalty term. Other possible approaches include the so-called monotone schemes such as upwind procedures or streamline upwind Petrov-Galerkin methods [32].

Contributions more closely related to the present paper include the work of Guardone and Vigevano [35], who present a "node-pair" FE method and make the link with the FV approximation for axially symmetric scalar conservation laws. Kleine and Reddy [37] study different geometries for settling tanks using a FE method, while Calgaro, Creusé, and Goudon [16] couple Taylor-Hood finite elements for the flow equations with finite volumes for a conservation law.

The reduction of three-dimensional problems to axisymmetric ones is addressed in the early work by Mercier and Raugel [42] (for Poisson problems) and by Belhachmi, Bernardi, and Deparis [3] and Belhachmi et al. [4] for the Stokes and Navier-Stokes equations in the primitive variables and by Carneiro de Araujo and Ruas [17] for a three-field formulation.

Models similar to (1.1) also arise in other applications, including so-called timedependent natural convection flows, in which the Navier-Stokes equations are coupled with a transport equation for temperature [21], thermal convection flows [52], the melting of glaciers [36], aluminum production [31], petroleum reservoir engineering [50], and nuclear waste contamination [20]. The present numerical method should therefore be of interest in these applications. 
1.3. Outline of the paper. The remainder of this paper is organized as follows. In section 2, some preliminaries about notation and the model itself along with its weak formulation and a discussion on its solvability are presented. In section 3 we derive an FVE method for the numerical approximation of the weak formulation. To this end we first introduce in section 3.1 the appropriate FE spaces for the approximation of $\phi, \boldsymbol{u}$, and $p$ in an axisymmetric setting. Then we formulate the semidiscrete Galerkin discretizations for the axisymmetric versions of the convectiondiffusion equation (1.1a) and the Stokes system (1.1b), (1.1c), respectively (in section 3.2). Then, in section 3.3, we proceed to the formulation of the FVE method for the coupled problem, and we specify in section 3.4 the space-time-discrete scheme. Section 4 provides several numerical experiments to illustrate the performance of the method and the applicability of the model, and we close in section 5 with some conclusions and comments on current extensions.

\section{Preliminaries and statement of the problem.}

2.1. Notation. Let $\mathcal{R}$ denote an open, bounded, and connected subset of $\mathbb{R}^{d}$, $d=2,3$, with a piecewise smooth boundary $\partial \mathcal{R}$. We use standard notation for Lebesgue spaces $L^{p}(\mathcal{R}), 1 \leq p \leq \infty, L_{0}^{2}(\mathcal{R})=\left\{v \in L^{2}(\mathcal{R}): \int_{\mathcal{R}} v \mathrm{~d} \boldsymbol{x}=0\right\}$, and Sobolev functional spaces $H^{m}(\mathcal{R}), m \geq 0$. In particular $L^{2}(R)=H^{0}(R)$. We also write $H_{0}^{1}(\mathcal{R})=\left\{v \in H^{1}(\mathcal{R}): v=0\right.$ on $\left.\partial \mathcal{R}\right\}$, and by $\boldsymbol{n}$ we denote the outward normal vector to $\partial \mathcal{R}$. For $T>0$, standard Bochner spaces are denoted by $L^{p}\left(0, T ; H^{m}(\mathcal{R})\right)$. To distinguish between scalar and vector functions, we will use boldface symbols for vector functions. Similarly, a function space written in boldface denotes the vector analogue of the corresponding space of scalar functions.

2.2. Axisymmetric formulation. We are interested in solving (1.1) in a domain $\tilde{\Omega} \subset \mathbb{R}^{3}$ which is assumed to be invariant by rotation around a vertical axis (see Figure 1(a)). To this end, we rewrite (1.1) in cylindrical coordinates $(r, \theta, z)$. Furthermore, $\Omega$ denotes the half cross section defined by $(r, 0, z)$ (see Figure $1(\mathrm{~b})$ ), on which we want to solve the problem. Since an axisymmetric scalar function $\tilde{s}$ defined on $\tilde{\Omega}$ depends only on $r$ and $z$, it is possible to associate to $\tilde{s}$ a function $s$ defined on $\Omega$ such that $s(r, z)=\tilde{s}(r, 0, z)$. If a vector field $\tilde{\boldsymbol{v}}$ defined on $\tilde{\Omega}$ has zero angular component, then a vector field $\boldsymbol{v}=\left(v_{r}, v_{z}\right)$ can be associated to it such that $\tilde{v}_{r}=v_{r}$, and $\tilde{v}_{z}=v_{z}$. This assumption corresponds to a so-called nonswirling flow regime, in which the datum and initial conditions have zero angular component [6]. In this context, the operators $\nabla_{\mathbf{a}}, \nabla_{\mathbf{a}} \cdot$ and the tensor $\varepsilon_{\mathbf{a}}$ are defined by

$$
\nabla_{\mathbf{a}} \boldsymbol{v}:=\left[\begin{array}{ll}
\partial_{r} v_{r} & \partial_{r} v_{z} \\
\partial_{z} v_{r} & \partial_{z} v_{z}
\end{array}\right], \quad \nabla_{\mathbf{a}} \cdot \boldsymbol{v}:=\partial_{z} v_{z}+\frac{1}{r} \partial_{r}\left(r v_{r}\right), \quad \boldsymbol{\varepsilon}_{\mathbf{a}}(\boldsymbol{v}):=\frac{1}{2}\left(\nabla_{\mathbf{a}} \boldsymbol{v}+\nabla_{\mathbf{a}} \boldsymbol{v}^{\mathrm{T}}\right),
$$

and for a scalar field $s$ we write $\nabla_{\mathbf{a}} s=\left(\partial_{r} s, \partial_{z} s\right)^{\mathrm{T}}$ and $\Delta_{\mathbf{a}} s=r^{-1} \partial_{r}\left(r \partial_{r} s\right)+\partial_{z}^{2} s$. Then, the nondimensional governing equations read

$$
\begin{aligned}
\partial_{t} \phi+\nabla_{\mathbf{a}} \cdot \boldsymbol{F}(\phi, \boldsymbol{u}) & =\Delta_{\mathbf{a}}(A(\phi)) \quad \text { in } \Omega, \quad t>0, \\
-\nabla_{\mathbf{a}} \cdot\left(\mu(\phi) \boldsymbol{\varepsilon}_{\mathbf{a}}(\boldsymbol{u})\right)+\lambda \nabla_{\mathbf{a}} p & =\boldsymbol{G}(\phi) \quad \text { in } \Omega, \\
\lambda \nabla_{\mathbf{a}} \cdot \boldsymbol{u} & =0 \quad \text { in } \Omega .
\end{aligned}
$$

As pointed out in $[6,17]$, to ensure that the model obtained by rewriting the original problem as (2.1)-(2.3) is consistent, we need to suppose axisymmetry not only of the domain but also of the relevant phenomenon under study. This means, in particular, that the flow spreads radially through the domain $\tilde{\Omega}$. 
(a)

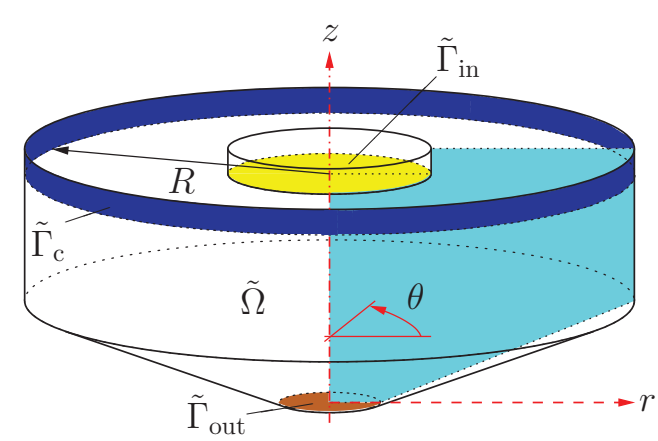

(b)

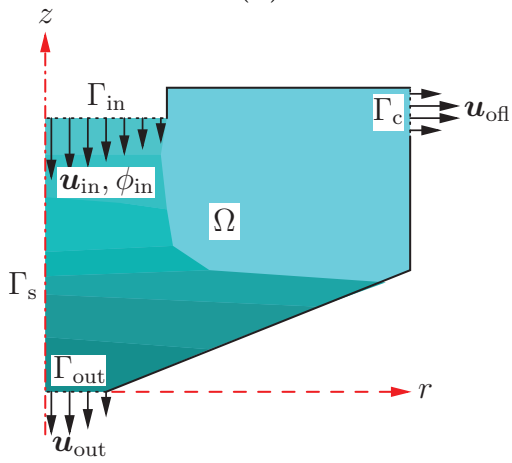

FIG. 1. (a) Sketch of a three-dimensional simplified clarifier-thickener unit $\tilde{\Omega}$. Here $z$ represents a symmetry axis. The disks $\tilde{\Gamma}_{\mathrm{in}}$ and $\tilde{\Gamma}_{\mathrm{out}}$ and the cylindrical strip $\tilde{\Gamma}_{\mathrm{c}}$ are the respective inflow, underflow, and overflow portions of the boundary $\partial \tilde{\Omega}$ of $\tilde{\Omega}$. (b) Schematic view of the axisymmetric domain $\Omega$. The boundary $\Gamma_{\mathrm{s}}$ represents the symmetry axis $r=0, \Gamma_{\mathrm{in}}$ is the inflow, $\Gamma_{\mathrm{out}}$ is the underflow, and $\Gamma_{\mathrm{c}}$ is the overflow boundary. In real-world applications, the bottom is of conical shape (as shown here) for ease of discharging sediment through the underflow opening, and the material (usually clear liquid) leaving through the overflow is collected in a circumferential open channel (not modeled or depicted here).

The field variables are the local volume fraction of solids (in short, concentration) $\phi=\phi(r, z, t)>0$, the volume average flow velocity of the mixture (in short, velocity) $\boldsymbol{u}=\boldsymbol{u}(r, z) \in \mathbb{R}^{2}$, and the total pressure $p=p(r, z) \in \mathbb{R}$. Clearly, $\boldsymbol{u}$ and $p$ also depend on time, but we drop this explicit dependence to emphasize that the same Stokes problem (2.2), (2.3) is solved for each $t>0$. As usual, (2.3) implies that the bulk suspension is regarded as an incompressible fluid.

2.3. Flux vector, diffusion term, viscosity, and body force. The flux vector in (2.1) is given by $\boldsymbol{F}(\phi, \boldsymbol{u})=\phi \boldsymbol{u}+f_{\mathrm{bk}}(\phi) \boldsymbol{k}$ (cf. [12]), where $\boldsymbol{k}$ is the unit vector pointing in the direction of gravity and $f_{\mathrm{bk}}$ is the Kynch batch flux density function [38] describing hindered settling. This function is assumed to satisfy $f_{\mathrm{bk}}(0)=$ $f_{\mathrm{bk}}\left(\phi_{\max }\right)=0$ and $f_{\mathrm{bk}}(\phi)>0$ for $0<\phi<\phi_{\max }$, where $0<\phi_{\max } \leq 1$ is a maximum concentration. Specifically, we choose here the expression [45]

$$
f_{\mathrm{bk}}(\phi)=\left\{\begin{array}{ll}
u_{\infty} \phi\left(1-\phi / \phi_{\max }\right)^{\mathrm{n}_{\mathrm{MB}}} & \text { for } 0<\phi<\phi_{\max }, \\
0 & \text { otherwise, }
\end{array} n_{\mathrm{MB}} \geq 1,\right.
$$

where $u_{\infty}>0$ is the settling velocity of a single particle in an unbounded fluid, and we choose the exponent $n_{\mathrm{MB}}=2$.

The term $\Delta A(\phi)$ models sediment compressibility, where the integrated diffusion function $A$ is given by

$$
A(\phi)=\int_{0}^{\phi} a(s) \mathrm{d} s, \quad \text { where } \quad a(\phi):=\frac{f_{\mathrm{bk}}(\phi) \sigma_{\mathrm{e}}^{\prime}(\phi)}{\left(\varrho_{\mathrm{s}}-\varrho_{\mathrm{f}}\right) g \phi} .
$$

Here $\varrho_{\mathrm{s}}$ and $\varrho_{\mathrm{f}}$ are the solid and fluid mass densities, respectively, $g$ is the acceleration of gravity, and $\sigma_{\mathrm{e}}^{\prime}$ is the derivative of effective solid stress function, $\sigma_{\mathrm{e}}$, which is here assumed to satisfy

$$
\sigma_{\mathrm{e}}^{\prime}(0)=0, \quad \sigma_{\mathrm{e}}^{\prime}(\phi)>0 \quad \text { for } \phi>0 .
$$


Since $a(0)=a\left(\phi_{\max }\right)=0$ and $a(\phi)>0$ for $0<\phi<\phi_{\max }$, (2.1) is a two-point degenerate parabolic PDE, which degenerates into a first-order hyperbolic conservation law for $\phi=0$ and $\phi=\phi_{\max }$. If in addition $a(\cdot)$ is assumed to be continuous, then in particular

$$
\Delta_{\mathbf{a}}(A(\phi))=\nabla_{\mathbf{a}} \cdot\left(a(\phi) \nabla_{\mathbf{a}} \phi\right) \quad \text { for } \phi \in(0,1]
$$

which is a useful asset in developing the numerical method.

Remark 2.1. It is frequently assumed that $\sigma_{\mathrm{e}}^{\prime}(\phi)=0$ not only for $\phi=0$ but for $0 \leq \phi \leq \phi_{\mathrm{c}}$, where $0<\phi_{\mathrm{c}}<\phi_{\max }$ is a critical concentration. In that case, (2.1) is strongly degenerate, and the well-posedness of this equation requires an entropy solution concept. Even though the development of our method is based on twopoint degeneracy, numerical experiments demonstrate that it handles the strongly degenerate case as well; see section 4.3.

The forcing term $\boldsymbol{G}(\phi)$ in (2.2) models that the mixture flow is driven by local fluctuations of $\phi$, and therefore of the density of the mixture, besides the inflow and discharge boundary conditions. More specifically, we put $\boldsymbol{G}(\phi)=-\lambda \phi g \boldsymbol{k}$.

Finally, $\mu(\phi)$ denotes a generalized local concentration-dependent Newtonian viscosity function, where we assume that there exist constants $\mu_{\min }, \mu_{\max }>0$ such that

$$
\mu_{\min }<\mu(s)<\mu_{\max } \quad \text { for } s \in \mathbb{R}_{+} .
$$

A suitable choice is $\mu(\phi)=\left(1-\phi / \tilde{\phi}_{\max }\right)^{-\beta}$ [44], where the parameter $\tilde{\phi}_{\max }$ is a second (nominal) maximum concentration. If we set $\tilde{\phi}_{\max }>\phi_{\max }$, then (2.5) is indeed valid.

2.4. Initial and boundary conditions. We are interested in solving (2.1)(2.3) for $t \in[0, T]$ in the spatial domain $\Omega$, which represents the axially symmetric cross section of the clarifier-thickener unit (see Figure 1(b)). The model is complemented by initial data for the concentration $\phi$ and the velocity $\boldsymbol{u}$, and boundary conditions are set as follows. The vessel is continuously fed through the inflow boundary $\Gamma_{\text {in }}$ with feed suspension, which corresponds to a given profile for the velocity $\boldsymbol{u}_{\text {in }}$, and a feed concentration $\phi_{\text {in }}$. On $\Gamma_{\text {out }}$ we prescribe a volume underflow velocity $\boldsymbol{u}_{\text {out }}$ at which the thickened sediment is removed from the unit and zero-flux conditions are assumed for $\phi$. On the symmetry axis (interior of the intersection of the whole boundary with the axis represented by $r=0$ ) denoted by $\Gamma_{\mathrm{s}}$ we set zero velocity in its perpendicular direction, and we assume that the flux component normal to the symmetry axis vanishes, i.e., $\left(\boldsymbol{F}(\phi, \boldsymbol{u})-a(\phi) \nabla_{\mathbf{a}} \phi\right) \cdot \boldsymbol{n}=0$ a.e. on $\Gamma_{\mathbf{s}}$.

On the remaining part of $\partial \Omega$ we specify no-slip boundary data for the velocity field $(\boldsymbol{u}=\mathbf{0})$ and zero-flux boundary conditions for the concentration. The datum $\boldsymbol{u}=\boldsymbol{u}_{\mathrm{D}} \in L^{2}(\Gamma)$ on $\Gamma=\partial \Omega$ summarizes all boundary conditions for velocity, whose specifications depend on the particular example and will be made precise in section 4 .

2.5. Weak solutions. To derive a weak form of (2.1)-(2.3), we recall the definitions of some weighted Lebesgue and Sobolev spaces (see, e.g., [4]). For $\alpha \in \mathbb{R}$ and $1 \leq p<\infty$, let $L_{\alpha}^{p}(\Omega)$ denote the space of measurable functions $v$ on $\Omega$ such that

$$
\|v\|_{L_{\alpha}^{p}(\Omega)}^{p}:=\int_{\Omega}|v|^{p} r^{\alpha} \mathrm{d} r \mathrm{~d} z \leq \infty .
$$

Obviously, for $p=\infty, L_{\alpha}^{\infty}(\Omega)$ coincides with $L^{\infty}(\Omega)$. We also use the space

$$
L_{1,0}^{2}(\Omega):=\left\{q \in L_{1}^{2}(\Omega): \int_{\Omega} q r \mathrm{~d} r \mathrm{~d} z=0\right\} .
$$


The scalar product in $L_{\alpha}^{2}(\Omega)$ is denoted by $(\cdot, \cdot)_{\alpha, \Omega}$. Moreover, $H_{\alpha}^{m}(\Omega)$ is the space of functions in $L_{\alpha}^{p}(\Omega)$ whose derivatives up to order $m$ are also in $L_{\alpha}^{p}(\Omega)$, and we denote by $H_{\alpha, 0}^{m}(\Omega)$ its restriction to functions with null trace on $\Gamma$. Its counterpart for general (nonhomogeneous) Dirichlet data is denoted $H_{\alpha, \Gamma}^{m}(\Omega)$. Finally, we introduce the spaces

$$
\begin{gathered}
V_{1}^{1}(\Omega):=\left\{w \in H_{1}^{1}(\Omega): w \in L_{-1}^{2}(\Omega)\right\}, \quad V_{1, \Gamma_{\mathrm{s}}}^{1}(\Omega):=\left\{w \in V_{1}^{1}(\Omega): w=0 \text { on } \Gamma_{\mathrm{s}}\right\} \\
S:=H_{1}^{1}(\Omega), \quad \mathcal{V}:=V_{1, \Gamma_{\mathrm{s}}}^{1}(\Omega) \times H_{1, \Gamma}^{1}(\Omega)
\end{gathered}
$$

The space of axisymmetric vector fields in $\left[H^{1}(\tilde{\Omega})\right]^{3}$ with zero angular component is isomorphic to $V_{1}^{1}(\Omega) \times H_{1}^{1}(\Omega)$ and the space of axisymmetric vector fields in $\left[H_{\Gamma}^{1}(\tilde{\Omega})\right]^{3}$ with zero angular component is isomorphic to $\mathcal{V}$ (see, e.g., [3]).

Multiplying (2.1), (2.2), and (2.3) by $r$ and smooth functions $s, \boldsymbol{v}$, and $q$, respectively, and integrating by parts over $\Omega$, which means that for any function $b \in L^{2}(\Omega)$,

$-\int_{\Omega} \nabla_{\mathbf{a}} \cdot\left(b \nabla_{\mathbf{a}} \boldsymbol{u}\right) \cdot \boldsymbol{v} r \mathrm{~d} r \mathrm{~d} z=\int_{\Omega}\left(b \nabla_{\mathbf{a}} \boldsymbol{u}:\left(\nabla_{\mathbf{a}} \boldsymbol{v}\right) r+\frac{b}{r} u_{r} v_{r}\right) \mathrm{d} r \mathrm{~d} z$ for $\boldsymbol{u}, \boldsymbol{v} \in H_{1,0}^{1}(\Omega)$,

yields the following weak formulation in the axisymmetric case: For $0<t<T$, find $(\phi(t), \boldsymbol{u}, p)=\left(\phi(t), \boldsymbol{u}_{\mathrm{D}}+\tilde{\boldsymbol{u}}, p\right) \in S \times \mathcal{V} \times Q$, with $\tilde{\boldsymbol{u}} \in H_{1,0}^{1}(\Omega)$, such that

$$
\begin{gathered}
\int_{\Omega} s \partial_{t} \phi r \mathrm{~d} r \mathrm{~d} z-\int_{\Omega}\left(\boldsymbol{F}(\phi, \boldsymbol{u})-a(\phi) \nabla_{\mathbf{a}} \phi\right) \cdot \nabla_{\mathbf{a}} s r \mathrm{~d} r \mathrm{~d} z+\int_{\Gamma_{\mathrm{in}}} r \phi s \mathrm{~d} \sigma \\
+\int_{\Gamma_{\mathrm{in}}} r s\left(\boldsymbol{F}(\phi, \boldsymbol{u})-a(\phi) \nabla_{\mathbf{a}} \phi\right) \cdot \boldsymbol{n} \mathrm{d} \sigma=\int_{\Gamma_{\mathrm{in}}} r \phi_{\mathrm{in}} s \mathrm{~d} \sigma \quad \forall s \in S, \\
\int_{\Omega} \mu(\phi) \boldsymbol{\varepsilon}_{\mathbf{a}}(\boldsymbol{u}): \boldsymbol{\varepsilon}_{\mathbf{a}}(\boldsymbol{v}) r \mathrm{~d} r \mathrm{~d} z+\int_{\Omega} \frac{1}{r} \mu(\phi) u_{r} v_{r} \mathrm{~d} r \mathrm{~d} z \\
\quad-\lambda \int_{\Omega} p \nabla_{\mathbf{a}} \cdot \boldsymbol{v} r \mathrm{~d} r \mathrm{~d} z=\int_{\Omega} \boldsymbol{G}(\phi) \cdot \boldsymbol{v} r \mathrm{~d} r \mathrm{~d} z \quad \forall \boldsymbol{v} \in H_{1,0}^{1}(\Omega), \\
\lambda \int_{\Omega} q \nabla_{\mathbf{a}} \cdot \boldsymbol{u} r \mathrm{~d} r \mathrm{~d} z=0 \quad \forall q \in Q
\end{gathered}
$$

and $\phi(0)=\phi_{0}$ a.e. in $\Omega$. Notice that the boundary condition $\left.\phi\right|_{\Gamma_{\text {in }}}=\phi_{\text {in }}$ is imposed weakly in the first equation of (2.6), whereas the condition $\left.\boldsymbol{u}\right|_{\partial \Omega}=\boldsymbol{u}_{\mathrm{D}}$ is included by means of a lifting.

Lemma 2.2. Let $0 \leq \phi_{0} \leq \phi_{\max }, \phi_{0} \in L^{\infty}(\Omega)$, and assume that $A(s) \in$ $L^{2}\left(0, T ; H_{1}^{1}(\Omega)\right)$ for $s \in S$ and $A\left(\phi_{\text {in }}\right) \in L^{2}\left(0, T ; H^{1 / 2}\left(\Gamma_{\text {in }}\right)\right)$. Then, there exists a unique solution to $(2.6)$, satisfying $\phi(0)=\phi_{0}, \phi \in L^{2}\left(0, T ; H_{1}^{1}(\Omega)\right) \cap C\left([0, T] ; L_{1}^{2}(\Omega)\right)$, and $\partial_{t} \phi \in L^{2}\left(0, T ;\left(H_{1}^{1}(\Omega)\right)^{\prime}\right)$.

A proof of Lemma 2.2 can be developed using the following sketch. First, the following closed subset of $L^{2}\left(0, T ; L_{1}^{2}(\Omega)\right)$ is considered:

$$
\mathcal{W}:=\left\{\phi \in L^{2}\left(0, T ; L_{1}^{2}(\Omega)\right): 0 \leq \phi(r, z, t) \leq \phi_{\max } \text { for a.e. }(r, z) \in \Omega, t>0\right\},
$$

i.e., a maximum principle is assumed for $\phi$. In the following step, the degeneracy of $a(\cdot)$ at $\phi=0$ is treated by defining an auxiliary system related to the term $a_{\varepsilon}(\phi)=a(\phi)+\varepsilon$ with $\varepsilon>0$. We then fix $\phi=\bar{\phi} \in \mathcal{W}$. The coupling terms $\mu(\bar{\phi})$ and $\boldsymbol{G}(\bar{\phi})$ are 
then uniformly bounded, which implies that the usual bilinear form associated to the diffusive term in (2.2) is continuous and coercive in $\mathcal{V}$ intersected with the space of (axisymmetric) divergence-free functions, and hence by the Lax-Milgram lemma we immediately obtain existence and uniqueness of solution $\boldsymbol{u}$. A unique pressure $p \in Q$ follows from the well-known Babuška-Brezzi condition satisfied for the pair $\mathcal{V} \times Q$. As a corollary we obtain that for a fixed $t>0$, the mentioned solution satisfies an estimate of the form $\|\boldsymbol{u}\|_{\mathcal{V}}+\|p\|_{Q} \leq C$, where $C$ depends only on $\|\bar{\phi}\|_{S}, \mu_{\max }$, and $|\Omega|$. Next, we fix $(\boldsymbol{u}, p)=(\overline{\boldsymbol{u}}, \bar{p})$ as the unique solution of $(2.2),(2.3)$. The forms of $a_{\varepsilon}(\cdot)$ and $\boldsymbol{F}(\cdot, \overline{\boldsymbol{u}})$, along with a classical result from [39], allow us to state the unique solvability of the auxiliary system related to the uniformly parabolic quasi-linear problem (2.1) for a fixed $(\overline{\boldsymbol{u}}, \bar{p})$ with boundary data $\phi=\phi_{\text {in }}$ on $\Gamma_{\text {in }}$, zero normal flux on $\Gamma_{\mathrm{s}}$, and zero-fluxes elsewhere (depending on $\overline{\boldsymbol{u}}$ ). Next, a solution map $\Theta: \mathcal{W} \rightarrow \mathcal{W}$ is defined such that $\Theta(\bar{\phi})=\phi$, i.e., $\phi$ is regarded as the solution of $(2.1)$ associated to $(\overline{\boldsymbol{u}}, \bar{p})$. This operator is well defined (in the sense that the maximum principle implied by $\mathcal{W}$ holds for $\Theta(\bar{\phi})$ ) and we can prove its continuity and compactness by demonstrating that sequences of regularized solutions satisfy a comparison principle, are bounded in $L^{\infty}\left(0, T ; L_{1}^{2}(\Omega)\right)$, and are relatively compact in $L^{2}\left(0, T ; L_{1}^{2}(\Omega)\right)$. Finally, by the Schauder fixed-point theorem one concludes that it admits a fixed point. The proof is complete after taking the limit $\varepsilon \rightarrow 0$.

\section{Approximation by finite volume elements.}

3.1. Axisymmetric FE setting. In what follows, $\mathcal{T}_{h}$ denotes a locally regular partition of the polygonal domain $\Omega$ into triangles $K$ of diameter $h_{K}$, that is, we assume that

$$
\exists C>0: \quad \forall K \in \mathcal{T}_{h}: \quad C h_{K}^{2} \leq|K| \leq h_{K}^{2} .
$$

The level of refinement of $\mathcal{T}_{h}$ is defined by the mesh parameter $h:=\max _{K \in \mathcal{T}_{h}}\left\{h_{K}\right\}$, and $\mathcal{N}_{h}:=\left\{s_{j}: j=1, \ldots, N\right\}$ is the set of nodes of $\mathcal{T}_{h}$. By $\mathcal{E}_{h}$ we denote the set of edges or interelement boundaries of $\mathcal{T}_{h}$, while $\mathcal{E}_{h}^{\text {int }}$ will denote the edges of $\mathcal{T}_{h}$ that are not part of $\partial \Omega$. As usual, $C$ will denote a generic constant $C>0$ that is independent of $h$. For simplicity, meshes are kept fixed in time.

By $S_{h}, \mathcal{V}_{h}$, and $Q_{h}$ we will denote the FE spaces for the approximation of $\phi, \boldsymbol{u}$, and $p$, respectively, which in our setting will be defined as follows:

$$
\begin{aligned}
S_{h} & :=\left\{s \in L_{1}^{2}(\Omega):\left.s\right|_{K} \in \mathbb{P}_{1}(K) \forall K \in \mathcal{T}_{h}\right\}, \\
\mathcal{V}_{h} & :=\left\{\boldsymbol{v} \in \mathcal{V} \cap C^{0}(\bar{\Omega}):\left.\boldsymbol{v}\right|_{K} \in \mathbb{P}_{1}(K)^{2} \forall K \in \mathcal{T}_{h}\right\}, \\
Q_{h} & :=\left\{q \in L_{1,0}^{2}(\Omega) \cap C^{0}(\bar{\Omega}):\left.q\right|_{K} \in \mathbb{P}_{1}(K) \forall K \in \mathcal{T}_{h}\right\} .
\end{aligned}
$$

Notice that Dirichlet data are imposed for $\boldsymbol{u}$ in the definition of $\mathcal{V}_{h}$. As usual, $\mathbb{P}_{m}(\mathcal{R})$ denotes the space of polynomial functions of total degree $s \leq m$ defined on the set $\mathcal{R}$ (in the coordinates $r$ and $z$ ). In addition, for a scalar function $w \in S$, we let $\llbracket w \rrbracket_{F}$ denote its jump and $\{w\}_{F}$ denote its mean value defined at the edge $F \in \mathcal{E}_{h}^{\text {int }}$ separating the neighboring elements $K^{+}$and $K^{-}$. That is,

$$
\llbracket w \rrbracket_{F}:=\left.w\right|_{K^{+}}-\left.w\right|_{K^{-}}, \quad\{w\}_{F}:=\frac{1}{2}\left(\left.w\right|_{K^{+}}+\left.w\right|_{K^{-}}\right) .
$$


For boundary edges $F \in \mathcal{E}_{h} \cap \partial \Omega$, these definitions simply reduce to

$$
\llbracket w \rrbracket_{F}:=\{w\}_{F}:=\left.w\right|_{F} .
$$

We also define the test space

$$
\mathcal{V}_{h}^{0}:=\left\{\boldsymbol{v} \in H_{1,0}^{1}(\Omega) \cap \boldsymbol{C}^{0}(\bar{\Omega}):\left.\boldsymbol{v}\right|_{K} \in \mathbb{P}_{1}(K)^{2} \forall K \in \mathcal{T}_{h}\right\} .
$$

Some approximation and interpolation properties associated to polynomial functions in weighted Sobolev spaces can be found in [6, Chapter V].

As is well known, the well-posedness of a Galerkin discretization for (2.1)-(2.3) will depend crucially on the choice of the finite-dimensional spaces. The discrete inf-sup condition states that there exists $\eta>0$ not depending on $h$ such that

$$
\sup _{\boldsymbol{v}_{h} \in \hat{V}_{h}} \frac{\int_{\Omega} \lambda q_{h} \nabla_{\mathbf{a}} \cdot \boldsymbol{v}_{h} \mathrm{~d} r \mathrm{~d} z}{\left\|\boldsymbol{v}_{h}\right\|_{H_{1}^{1}(\Omega)^{2}}} \geq \eta\left\|q_{h}\right\|_{L_{1}^{2}(\Omega)}, \quad q_{h} \in \hat{Q}_{h} .
$$

Our particular choice $\hat{V}_{h}=\mathcal{V}_{h}^{0}$ and $\hat{Q}_{h}=Q_{h}$ does not satisfy such a condition and therefore a stabilization method is needed (see section 3.2).

In what follows, starting from the weak formulation (2.6) we derive a Galerkin discretization of the coupled problem. Although the scheme in section 3.2 is not used for implementation, its derivation is useful to motivate the formulation of the final FVE scheme (in sections 3.3 and 3.4).

3.2. Galerkin FE formulation for the coupled problem. First, consider the following local semidiscrete Galerkin problem associated to (2.1) for a given element $K \in \mathcal{T}_{h}:$

$$
\begin{aligned}
& \frac{\mathrm{d}}{\mathrm{d} t} \int_{K} s_{h} \phi_{h}(t) r \mathrm{~d} r \mathrm{~d} z-\int_{K}\left(\boldsymbol{F}\left(\phi_{h}(t), \boldsymbol{u}_{h}\right)-a\left(\phi_{h}(t)\right) \nabla_{\mathbf{a}} \phi_{h}(t)\right) \cdot \nabla_{\mathbf{a}} s_{h} r \mathrm{~d} r \mathrm{~d} z \\
& \quad+\int_{\partial K} r s_{h}\left(\boldsymbol{F}\left(\phi_{h}(t), \boldsymbol{u}_{h}\right)-a\left(\phi_{h}(t)\right) \nabla_{\mathbf{a}} \phi_{h}(t)\right) \cdot \boldsymbol{n}_{K} \mathrm{~d} \sigma=0 \quad \forall s_{h} \in S_{h},
\end{aligned}
$$

where no boundary data have been considered yet. By the choice of the discrete space $S_{h}$ we do not impose continuity across interelement boundaries, and for such a discontinuous representation of functions of $S_{h}$, two values of the unknown $\phi_{h}(t)$ exist over a given edge $F \subset \partial K$. To handle this situation, the discontinuous boundary flux term is replaced by the Lax-Friedrichs numerical flux across $F \in \mathcal{E}_{h}$ defined by

$$
\widehat{\boldsymbol{F}}\left(\phi_{h}(t), \boldsymbol{u}_{h}\right) \cdot \boldsymbol{n}_{F}= \begin{cases}\left\{\boldsymbol{F}\left(\phi_{h}(t), \boldsymbol{u}_{h}\right)\right\}_{F} \cdot \boldsymbol{n}_{F}-\sigma_{F}^{*} \llbracket \phi_{h}(t) \rrbracket & \text { if } F \in \mathcal{E}_{h}^{\mathrm{int}}, \\ \boldsymbol{F}\left(\phi_{\mathrm{in}}, \boldsymbol{u}_{h}\right) \cdot \boldsymbol{n}_{F} & \text { if } F \subset \Gamma_{\mathrm{in}}, \\ \boldsymbol{F}\left(\phi_{h}(t), \boldsymbol{u}_{h}\right) \cdot \boldsymbol{n}_{F} & \text { otherwise, }\end{cases}
$$

where we define (cf., e.g., [22])

$$
\sigma_{F}^{*}:=\left(\boldsymbol{u}+\max _{0<\phi<\phi_{\max }} f_{\mathrm{bk}}^{\prime}(\phi) \boldsymbol{k}\right) \cdot \boldsymbol{n}_{F} .
$$

In addition, the Dirichlet boundary datum $\phi_{h}=\phi_{\text {in }}$ on $\Gamma_{\text {in }}$ is imposed weakly (see, e.g., $[25,34])$ and a general stabilization procedure is applied, where the additional terms are similar to those in $[13,22]$. 
Next, the discretization of (2.2), (2.3) follows the lines of [48] in the context of the well-known variational multiscale methods $[2,32]$. In short, the trial functional space for $\boldsymbol{u}$ is enriched with a space of functions that do not vanish on the element boundaries and which are split into a bubble part and a harmonic extension of the local boundary condition. After a static condensation procedure, the enriched part is completely identified, and so the original discrete problem can be recast as a stabilized method in terms of classical piecewise linear elements for both velocity and pressure.

Summarizing, we may state the proposed Galerkin FE formulation associated to the weak formulation (2.6) of the coupled problem (2.1)-(2.3) as follows. For $t \in(0, T]$, find $\left(\phi_{h}(t), \boldsymbol{u}_{h}, p_{h}\right) \in S_{h} \times \mathcal{V}_{h} \times Q_{h}$ such that

$$
\begin{aligned}
& \frac{\mathrm{d}}{\mathrm{d} t}\left(\phi_{h}(t), s_{h}\right)_{1, \Omega}-\left(\boldsymbol{F}\left(\phi_{h}(t), \boldsymbol{u}_{h}\right)-a\left(\phi_{h}(t)\right) \nabla_{\mathbf{a}} \phi_{h}(t), \nabla_{\mathbf{a}} s_{h}\right)_{1, \Omega} \\
& +\sum_{F \in \mathcal{E}_{h}^{\text {int }}}\left(\widehat{\boldsymbol{F}}\left(\phi_{h}(t), \boldsymbol{u}_{h}\right) \cdot \boldsymbol{n}_{F}-\left\{a\left(\phi_{h}(t)\right) \nabla_{\mathbf{a}} \phi_{h}(t) \cdot \boldsymbol{n}_{F}\right\}_{F}+\frac{\kappa_{F}^{\phi}}{h_{F}^{2}} \llbracket \phi_{h}(t) \rrbracket_{F}, \llbracket s_{h} \rrbracket\right)_{1, F} \\
& -\eta \sum_{F \in \mathcal{E}_{h}^{\text {int }}}\left(\llbracket \phi_{h}(t) \rrbracket_{F},\left\{a\left(\phi_{h}(t)\right) \nabla_{\mathbf{a}} s_{h} \cdot \boldsymbol{n}_{F}\right\}_{F}\right)_{1, F}+\sum_{F \subset \partial K \cap \Gamma_{\text {in }}}\left(\frac{\kappa_{F}^{\phi}}{h_{F}^{2}} \phi_{h}(t), s_{h}\right)_{1, F} \\
& -\sum_{F \in \mathcal{E}_{h} \cap \Gamma_{\text {in }}}\left[\eta\left(\phi_{\text {in }}, a\left(\phi_{h}(t)\right) \nabla_{\mathbf{a}} s_{h} \cdot \boldsymbol{n}_{F}\right)_{1, F}-\left(\frac{\kappa_{F}^{\phi}}{h_{F}^{2}} \phi_{\text {in }}, s_{h}\right)_{1, F}\right]=0 \quad \forall s_{h} \in S_{h}, \\
& \left(\mu\left(\phi_{h}(t)\right) \varepsilon_{\mathbf{a}}\left(\boldsymbol{u}_{h}\right), \boldsymbol{\varepsilon}_{\mathbf{a}}\left(\boldsymbol{v}_{h}\right)\right)_{1, \Omega}+\left(\mu\left(\phi_{h}(t)\right) u_{h, r}, v_{h, r}\right)_{-1, \Omega} \\
& \left.-\lambda\left(p_{h}, \nabla_{\mathbf{a}} \cdot \boldsymbol{v}_{h}\right)_{1, \Omega}-\left(\boldsymbol{G}_{\left(\phi_{h}\right.}(t)\right), \boldsymbol{v}_{h}\right)_{1, \Omega} \\
& +\sum_{F \in \mathcal{E}_{h}^{\text {int }}} \frac{h_{F}}{\kappa_{F}^{\boldsymbol{u}}\left(\llbracket \mu\left(\phi_{h}(t)\right) \partial_{\boldsymbol{n}_{F}} \boldsymbol{u}_{h} \rrbracket_{F}, \llbracket \mu\left(\phi_{h}(t)\right) \partial_{\boldsymbol{n}_{F}} \boldsymbol{v}_{h} \rrbracket_{F}\right)_{1, F}=0 \quad \forall \boldsymbol{v}_{h} \in \mathcal{V}_{h}^{0},} \\
& \lambda\left(q_{h}, \nabla_{\mathbf{a}} \cdot \boldsymbol{u}_{h}\right)_{1, \Omega}+\sum_{K \in \mathcal{T}_{h}} \frac{h_{K}^{2}}{\kappa_{K}^{p}}\left(\lambda \nabla_{\mathbf{a}} p_{h}-\boldsymbol{G}\left(\phi_{h}(t)\right), \nabla_{\mathbf{a}} q_{h}\right)_{1, K}=0 \quad \forall q_{h} \in Q_{h} .
\end{aligned}
$$

Here $h_{F}$ stands for the one-dimensional measure of $F, \kappa_{F}^{\phi}$ is a stabilization parameter (constant along $F$ ) for the concentration field, to be adjusted appropriately, and the parameter $\eta$ assumes the values $0,1,-1$ for the incomplete, symmetric, and nonsymmetric interior penalty Galerkin methods, respectively. The remaining stabilization parameters are chosen as $\kappa_{F}^{\boldsymbol{u}}=\mu_{\max }$ and $\kappa_{K}^{p}=2 \mu_{\max }$, and $w_{h, r}$ denotes the $r$ component of any discrete function $\boldsymbol{w}_{h}$. Edge-based discretizations of this type help enforcing the global mass conservation property (see [13]). At time $t=0$, the approximate concentration $\phi_{h}(0)$ is constructed by taking the $L^{2}$-orthogonal projection of $\phi_{0}$ onto $S_{h}$.

3.3. The FVE method. For the formulation of the FVE counterpart of (3.2), (3.3), we require the so-called dual partition $\mathcal{T}_{h}^{\star}$ of $\Omega$, which is defined based on the primal mesh $\mathcal{T}_{h}$ by constructing nonoverlapping control volumes $K_{i}^{\star}$ surrounding the node $s_{i} \in \mathcal{N}_{i}$ (as sketched in Figure 2). The control volumes can be introduced in several different ways, but we stick to barycentric dual meshes. In a given triangle $K \in \mathcal{T}_{h}$, we select its barycenter $b_{K}$ and create segments joining $b_{K}$ with the midpoints 


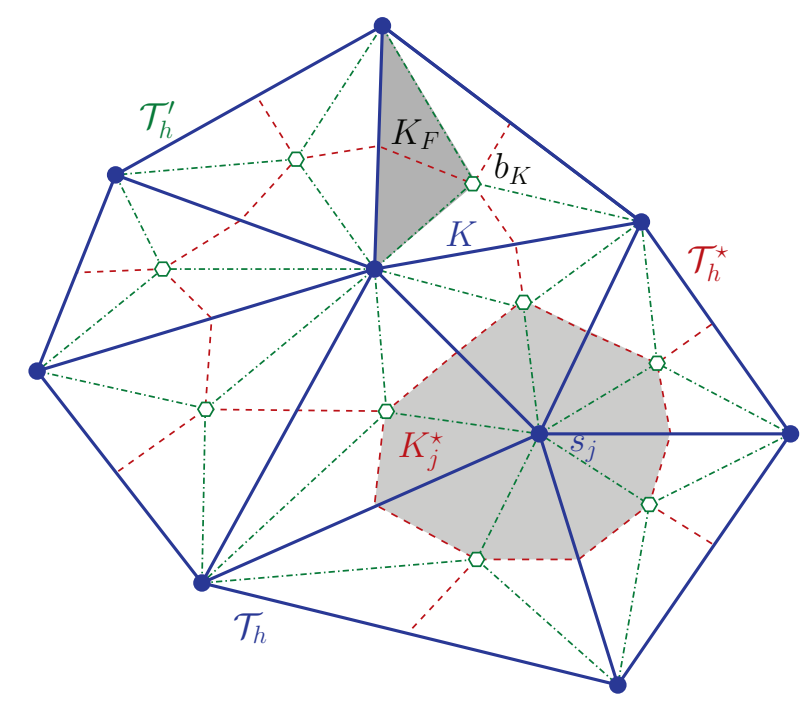

Fig. 2. Sketch of triangular elements in the primal mesh $\mathcal{T}_{h}$, interior node-centered control volumes of the dual mesh $\mathcal{T}_{h}^{\star}$ (in dashed lines), and triangular elements of the diamond mesh $\mathcal{T}_{h}^{\prime}$ (in dashed-dotted lines). A control volume $K_{j}^{\star}$ surrounding a node $s_{j}$, and an element $K_{F}$ of the diamond mesh are enhanced.

$m_{F}$ of each edge $F \subset \partial K$. This operation is performed for all elements in $K$, and in this way one control volume $K_{i}^{\star}$ will be associated to the vertex $s_{i}$, which is formed by all subelements sharing the vertex $s_{i}$.

Since in section 3.2 we approximate the $\phi$-field by discontinuous finite elements, a further mesh is needed for the FVE formulation associated to (3.1). We refer to that mesh as the diamond mesh (although it does not correspond exactly to the classical concept of diamond meshes arising in the construction of discrete duality FV methods, such as the one in [1]). As we will see later, both the dual and diamond meshes will be needed only for the formulation of the FVE scheme, but no longer at the implementation stage, since the terms containing the contributions on $\mathcal{T}_{h}^{\prime}$ are actually lying on the edges of $\mathcal{T}_{h}$. The diamond mesh $\mathcal{T}_{h}^{\prime}$ is constructed simply by joining the barycenter $b_{K}$ of the generic triangle $K \in \mathcal{T}_{h}$ with the vertices of $K$, forming in this way three subtriangles $K_{F}$, for $F \subset \partial K$, for a given $K \in \mathcal{T}_{h}$. We now define some finite-dimensional spaces associated to $\mathcal{T}_{h}^{\star}$ and $\mathcal{T}_{h}^{\prime}$. First, we define

$$
\begin{aligned}
\mathcal{V}_{h}^{\star}:=\left\{\boldsymbol{v} \in \boldsymbol{L}_{1}^{2}(\Omega):\left.\boldsymbol{v}\right|_{K_{j}^{\star}} \in \mathbb{P}_{0}\left(K_{j}^{\star}\right)^{2} \forall K_{j}^{\star} \in \mathcal{T}_{h}^{\star},\right. \\
\\
\left.\left.\boldsymbol{v}\right|_{K_{j}^{\star}}=\boldsymbol{u}_{\mathrm{D}} \text { if } K_{j}^{\star} \text { is a boundary volume }\right\},
\end{aligned}
$$

spanned by $\left\{\boldsymbol{\chi}_{i}(1,0), \boldsymbol{\chi}_{i}(0,1)\right\}_{i}$, where $\boldsymbol{\chi}_{i}$ is the characteristic function of the control volume $K_{i}^{\star}$, that is,

$$
\chi_{i}(x)= \begin{cases}1 & \text { if } x \in K_{i}^{\star} \\ 0 & \text { otherwise }\end{cases}
$$

and $\boldsymbol{u}_{\mathrm{D}}$ is a generic Dirichlet datum for velocity. Second, we introduce the space

$$
S_{h}^{\prime}:=\left\{s \in L_{1}^{2}(\Omega):\left.s\right|_{K_{F}} \in \mathbb{P}_{0}\left(K_{F}\right) \forall K_{F} \in \mathcal{T}_{h}^{\prime}\right\}
$$


Moreover, we introduce the transfer operator $\mathcal{P}: \mathcal{V}_{h} \rightarrow \mathcal{V}_{h}^{\star}$ (cf. [15, 48]), which for all $\boldsymbol{v}_{h} \in \mathcal{V}_{h}$ is defined as follows. If

$$
\boldsymbol{v}_{h}(x)=\sum_{i=1}^{N_{h}} \boldsymbol{v}_{h}\left(s_{i}\right) \phi_{i}(x) \quad \text { for } x \in \Omega,
$$

where $\left\{\boldsymbol{\phi}_{i}\right\}_{i}$ is the canonical FE basis of $\mathcal{V}_{h}$, then

$$
\left(\mathcal{P}_{h} \boldsymbol{v}_{h}\right)(x)=\sum_{i=1}^{N_{h}} \boldsymbol{v}_{h}\left(s_{i}\right) \boldsymbol{\chi}_{i}(x) \quad \text { for } x \in \Omega .
$$

We also define the bijective map $\mathcal{R}_{h}: S_{h} \rightarrow S_{h}^{\prime}$ by [7]

$$
\left.\mathcal{R}_{h} s_{h}\right|_{K_{F}}=\left.\frac{1}{h_{F}} \int_{F} s_{h}\right|_{K_{F}} \mathrm{~d} \sigma \quad \text { for } s_{h} \in S_{h}, K_{F} \in \mathcal{T}_{h}^{\prime} .
$$

The discrete problem associated to the variational formulation is obtained by multiplying (2.1) by $\mathcal{R}_{h} s_{h} \in S_{h}^{\prime}$, integrating by parts over each $K_{F} \in \mathcal{T}_{h}^{\prime}$, multiplying (2.2) by $\mathcal{P}_{h} \boldsymbol{v}_{h} \in \mathcal{V}_{h}^{\star}$ and integrating by parts over each control volume $K_{i}^{\star} \in \mathcal{T}_{h}^{\star}$, and multiplying (2.3) by $q_{h} \in Q_{h}$ and integrating by parts over each element $K \in \mathcal{T}_{h}$. This gives the following (unstable) semidiscrete Petrov-Galerkin interior formulation: For $0<t \leq T$, find $\phi_{h}(t) \in S_{h}, \boldsymbol{u}_{h} \in \mathcal{V}_{h}, p_{h} \in Q_{h}$ such that

$$
\begin{aligned}
& \frac{\mathrm{d}}{\mathrm{d} t}\left(\phi_{h}(t), s_{h}^{\prime}\right)_{1, \Omega}-\sum_{K \in \mathcal{T}_{h}} \sum_{K_{F} \subset K} \int_{\partial K_{F}} s_{h}^{\prime}\left(\boldsymbol{F}\left(\phi_{h}(t), \boldsymbol{u}_{h}\right)-a\left(\phi_{h}(t)\right)\right) \partial_{\boldsymbol{n}} \phi_{h}(t) r \mathrm{~d} \sigma=0, \\
& \quad-\sum_{i=1}^{N_{h}} \int_{\partial K_{i}^{\star}} \mu\left(\phi_{h}(t)\right) \boldsymbol{v}_{h}^{\star} \cdot \partial_{\boldsymbol{n}} \boldsymbol{u}_{h} r \mathrm{~d} \sigma-\lambda \sum_{i=1}^{N_{h}} \int_{\partial K_{i}^{\star}} p_{h} \boldsymbol{v}_{h}^{\star} \cdot \boldsymbol{n} r \mathrm{~d} \sigma=\left(\boldsymbol{G}\left(\phi_{h}(t)\right), \boldsymbol{v}_{h}^{\star}\right)_{1, \Omega}, \\
& \lambda \sum_{i=1}^{N_{h}} \boldsymbol{u}_{h}\left(s_{i}\right) \int_{\partial K_{i}^{\star}} q_{h} \boldsymbol{n} r \mathrm{~d} \sigma=0 \quad \forall s_{h}^{\prime} \in S_{h}^{\prime}, \boldsymbol{v}_{h}^{\star} \in \mathcal{V}_{h}^{\star}, q_{h} \in Q_{h} .
\end{aligned}
$$

To recast this formulation as a Galerkin method where the trial and test spaces coincide, we employ the next lemma.

Lemma 3.1. The following equations hold for all $\boldsymbol{w}_{h}, \boldsymbol{v}_{h} \in \mathcal{V}_{h}^{0}, q_{h} \in Q_{h}$, and $\xi_{h}, s_{h} \in S_{h}:$

$$
\begin{aligned}
& -\sum_{i=1}^{N_{h}} \boldsymbol{v}_{h}\left(s_{i}\right) \int_{\partial K_{i}^{\star}} \mu(r, z) \partial_{\boldsymbol{n}} \boldsymbol{u}_{h} r \mathrm{~d} \sigma \\
& \quad=\left(\mu(r, z) \boldsymbol{\varepsilon}_{\mathbf{a}}\left(\boldsymbol{u}_{h}\right), \boldsymbol{\varepsilon}_{\mathbf{a}}\left(\boldsymbol{v}_{h}\right)\right)_{1, \Omega}+\left(\mu(r, z) u_{h, r}, v_{h, r}\right)_{-1, \Omega}, \\
& \sum_{i=1}^{N_{h}} \boldsymbol{v}_{h}\left(s_{i}\right) \int_{\partial K_{i}^{\star}} q_{h} \boldsymbol{n} r \mathrm{~d} \sigma=\left(q_{h}, \nabla_{\mathbf{a}} \cdot \boldsymbol{v}_{h}\right)_{1, \Omega}, \\
& -\sum_{K \in \mathcal{T}_{h}} \sum_{K_{F} \subset K} \int_{\partial K_{F}} \mathcal{R}_{h} s_{h} a\left(\xi_{h}\right) \partial_{\boldsymbol{n}} \xi_{h} r \mathrm{~d} \sigma \\
& \quad=\left(a\left(\xi_{h}\right) \nabla_{\mathbf{a}} \xi_{h}, \nabla_{\mathbf{a}} s_{h}\right)_{1, \Omega}+\sum_{F \in \mathcal{E}_{h}^{\text {int }}}\left(a\left(\xi_{h}\right) \partial_{\boldsymbol{n}} \xi_{h}, \mathcal{R}_{h} s_{h}-s_{h}\right)_{1, F} .
\end{aligned}
$$


Proof. For (3.6) it suffices to apply [19, Lemma 2.2] noting the continuity of $a\left(\xi_{h}\right) \nabla_{\mathbf{a}} \xi_{h} \cdot \boldsymbol{n}$, whereas (3.4) and (3.5) follow as in [48].

In light of Lemma 3.1 we conclude that the semidiscrete stabilized FVE approximation of (2.1)-(2.3) (whose solution we will also denote by $\left.\phi_{h}, \boldsymbol{u}_{h}, p_{h}\right)$ is the solution of the following problem: For $0<t \leq T$, find $\phi_{h}(t) \in S_{h}, \boldsymbol{u}_{h} \in \mathcal{V}_{h}, p_{h} \in Q_{h}$ such that

$$
\begin{aligned}
& \frac{\mathrm{d}}{\mathrm{d} t}\left(\phi_{h}(t), \mathcal{R}_{h} s_{h}\right)_{1, \Omega}-\left(\boldsymbol{F}\left(\phi_{h}(t), \boldsymbol{u}_{h}\right)-a\left(\phi_{h}(t)\right) \nabla_{\mathbf{a}} \phi_{h}(t), \nabla_{\mathbf{a}} s_{h}\right)_{1, \Omega} \\
& +\sum_{F \in \mathcal{E}_{h}^{\text {int }}}\left(\widehat{\boldsymbol{F}}\left(\phi_{h}(t), \boldsymbol{u}_{h}\right) \cdot \boldsymbol{n}_{F}-\left\{a\left(\phi_{h}(t)\right) \nabla_{\mathbf{a}} \phi_{h}(t) \cdot \boldsymbol{n}_{F}\right\}_{F}+\frac{\kappa_{F}^{\phi}}{h_{F}^{2}} \llbracket \mathcal{R}_{h} \phi_{h}(t) \rrbracket_{F}, \llbracket \mathcal{R}_{h} s_{h} \rrbracket_{F}\right)_{1, F} \\
& -\eta \sum_{F \in \mathcal{E}_{h}^{\text {int }}}\left(\llbracket \phi_{h}(t) \rrbracket_{F},\left\{a\left(\phi_{h}(t)\right) \nabla_{\mathbf{a}} s_{h} \cdot \boldsymbol{n}_{F}\right\}_{F}\right)_{1, F}+\sum_{F \subset \partial K \cap \Gamma_{\text {in }}}\left(\frac{\kappa_{F}^{\phi}}{h_{F}^{2}} \phi_{h}(t), \mathcal{R}_{h} s_{h}\right)_{1, F} \\
& -\sum_{F \in \mathcal{E}_{h} \cap \Gamma_{\text {in }}}\left[\eta\left(\phi_{\text {in }}, a\left(\phi_{h}(t)\right) \nabla_{\mathbf{a}} s_{h} \cdot \boldsymbol{n}_{F}\right)_{1, F}-\left(\frac{\kappa_{F}^{\phi}}{h_{F}^{2}} \phi_{\text {in }}, \mathcal{R}_{h} s_{h}\right)_{1, F}\right]=0 \quad \forall s_{h} \in S_{h}, \\
& \left(\mu\left(\phi_{h}(t)\right) \boldsymbol{\varepsilon}_{\mathbf{a}}\left(\boldsymbol{u}_{h}\right), \boldsymbol{\varepsilon}_{\mathbf{a}}\left(\boldsymbol{v}_{h}\right)\right)_{1, \Omega}+\left(\mu\left(\phi_{h}(t)\right) u_{h, r}, v_{h, r}\right)_{-1, \Omega} \\
& -\lambda\left(p_{h}, \nabla_{\mathbf{a}} \cdot \boldsymbol{v}_{h}\right)_{1, \Omega}-\left(\boldsymbol{G}\left(\phi_{h}(t)\right), \mathcal{P}_{h} \boldsymbol{v}_{h}\right)_{1, \Omega} \\
& +\sum_{F \in \mathcal{E}_{h}^{\text {int }}} \frac{h_{F}}{\kappa_{F}^{\boldsymbol{u}}}\left(\llbracket \mu\left(\phi_{h}(t)\right) \boldsymbol{\varepsilon}_{\mathbf{a}}\left(\boldsymbol{u}_{h}\right) \cdot \boldsymbol{n}_{F} \rrbracket_{F}, \llbracket \mu\left(\phi_{h}(t)\right) \boldsymbol{\varepsilon}_{\mathbf{a}}\left(\boldsymbol{v}_{h}\right) \cdot \boldsymbol{n}_{F} \rrbracket_{F}\right)_{1, F}=0 \quad \forall \boldsymbol{v}_{h} \in \mathcal{V}_{h}^{0}, \\
& \lambda\left(q_{h}, \nabla_{\mathbf{a}} \cdot \boldsymbol{u}_{h}\right)_{1, \Omega}+\sum_{K \in \mathcal{T}_{h}} \frac{h_{K}^{2}}{\kappa_{K}^{p}}\left(\lambda \nabla_{\mathbf{a}} p_{h}-\boldsymbol{G}\left(\phi_{h}(t)\right), \nabla_{\mathbf{a}} q_{h}\right)_{1, K}=0 \quad \forall q_{h} \in Q_{h} .
\end{aligned}
$$

By virtue of Lemma 3.1 the two last schemes are equivalent up to stabilization terms that vanish asymptotically.

3.4. Space-time discrete scheme. We introduce a variable time step $\Delta t^{n}=$ $t^{n+1}-t^{n}$ and partition the time interval as $\left[0, \ldots, t^{n}, \ldots, T\right]$. Furthermore, by $w^{n}$ we denote the quantity $w\left(\cdot, t^{n}\right)$. Then, to advance the solution from $t^{n}$ to $t^{n+1}$ we solve the time discrete problem by the following splitting (or segregating) method (we here consider the incomplete interior penalty method):

1. Given $\phi_{h}^{n}$, compute $\boldsymbol{u}_{h}^{n}$ and $p_{h}^{n}$ by solving the Stokes problem

$$
\begin{aligned}
& \left(\mu\left(\phi_{h}^{n}\right) \boldsymbol{\varepsilon}_{\mathbf{a}}\left(\boldsymbol{u}_{h}^{n}\right), \boldsymbol{\varepsilon}_{\mathbf{a}}\left(\boldsymbol{v}_{h}\right)\right)_{1, \Omega}+\left(\mu\left(\phi_{h}^{n}\right) u_{h, r}^{n}, v_{h, r}\right)_{-1, \Omega} \\
& -\lambda\left(p_{h}^{n}, \nabla_{\mathbf{a}} \cdot \boldsymbol{v}_{h}\right)_{1, \Omega}-\left(\boldsymbol{G}\left(\phi_{h}^{n}\right), \mathcal{P}_{h} \boldsymbol{v}_{h}\right)_{1, \Omega} \\
& +\sum_{F \in \mathcal{E}_{h}^{\text {int }}} \frac{h_{F}}{\kappa_{F}^{\mathbf{u}}}\left(\llbracket \mu\left(\phi_{h}^{n}\right) \boldsymbol{\varepsilon}_{\mathbf{a}}\left(\boldsymbol{u}_{h}^{n}\right) \cdot \boldsymbol{n}_{F} \rrbracket_{F}, \llbracket \mu\left(\phi_{h}^{n}\right) \boldsymbol{\varepsilon}_{\mathbf{a}}\left(\boldsymbol{v}_{h}\right) \cdot \boldsymbol{n}_{F} \rrbracket_{F}\right)_{1, F}=0 \quad \forall \boldsymbol{v}_{h} \in \mathcal{V}_{h}^{0}, \\
& \lambda\left(q_{h}, \nabla_{\mathbf{a}} \cdot \boldsymbol{u}_{h}^{n}\right)_{1, \Omega}+\sum_{K \in \mathcal{T}_{h}} \frac{h_{K}^{2}}{\kappa_{K}^{p}}\left(\lambda \nabla_{\mathbf{a}} p_{h}^{n}-\boldsymbol{G}\left(\phi_{h}^{n}\right), \nabla_{\mathbf{a}} q_{h}\right)_{1, K}=0 \quad \forall q_{h} \in Q_{h} .
\end{aligned}
$$


2. Given $\boldsymbol{u}_{h}^{n}$ and $p_{h}^{n}$ computed in step 1, obtain $\phi_{h}^{n+1}$ from the following scheme associated to the parabolic problem:

$$
\begin{gathered}
\left(\frac{\phi_{h}^{n+1}-\phi_{h}^{n}}{\Delta t^{n}}, \mathcal{R}_{h} s_{h}\right)_{1, \Omega}-\left(\boldsymbol{F}\left(\phi_{h}^{n+1}, \boldsymbol{u}_{h}^{n}\right)-a\left(\phi_{h}^{n}\right) \nabla_{\mathbf{a}} \phi_{h}^{n+1}, \nabla_{\mathbf{a}} s_{h}\right)_{1, \Omega} \\
+\sum_{F \in \mathcal{E}_{h} \cap \Gamma_{\text {in }}}\left(\frac{\kappa_{F}^{\phi}}{h_{F}^{2}} \phi_{h}^{n+1}, \mathcal{R}_{h} s_{h}\right)_{1, F}+\left(\frac{\kappa_{F}^{\phi}}{h_{F}^{2}} \phi_{\text {in }}, \mathcal{R}_{h} s_{h}\right)_{1, F} \\
+\sum_{F \in \mathcal{E}_{h}^{\text {int }}}\left(\widehat{\boldsymbol{F}}\left(\phi_{h}^{n+1}, \boldsymbol{u}_{h}^{n}\right) \cdot \boldsymbol{n}_{F}-\left\{a\left(\phi_{h}^{n}\right) \nabla_{\mathbf{a}} \phi_{h}^{n+1} \cdot \boldsymbol{n}_{F}\right\}_{F}\right. \\
\left.+\frac{\kappa_{F}^{\phi}}{h_{F}^{2}} \llbracket \mathcal{R}_{h} \phi_{h}^{n+1} \rrbracket_{F}, \llbracket \mathcal{R}_{h} s_{h} \rrbracket_{F}\right)_{1, F}=0 \quad \forall s_{h} \in S_{h} .
\end{gathered}
$$

Computational efficiency is the principal motivation for our procedure to use a segregated solution algorithm to compute $\phi, \boldsymbol{u}$, and $p$. Furthermore, to reduce the complexity of the tangent system needed by Newton's method at every time step (we found that in almost every time step, fewer than 10 iterations are enough to achieve convergence), the time discretization (3.7) corresponds to a semi-implicit procedure (the terms $a\left(\phi_{h}\right)$ are taken in the previous time step), and so the nonlinearity is carried only by the term $\boldsymbol{F}\left(\cdot, \boldsymbol{u}_{h}^{n}\right)$. We also mention that the first term in the lefthand side of (3.7) could be replaced by $\frac{\mathrm{d}}{\mathrm{d} t}\left(\phi_{h}(t), s_{h}\right)_{1, \Omega}$ (as is done, e.g., in [51] in the context of the transient Stokes equations, with the objective of a classical convergence analysis). Notice that the stabilization terms for $\boldsymbol{u}$ and $\phi$ in the FVE formulation lead to a nonsymmetric linear system, which at each time step we solve by means of the unsymmetric multifrontal direct solver for sparse matrices (UMFPACK [23]).

4. Numerical results. We report in this section the results of three numerical tests computed with the proposed formulation. Before solving (2.1)-(2.3) for the clarifier-thickener unit, we consider in Example 1 a simple linear model problem (in Cartesian coordinates) with an explicit exact solution in order to test the (spatial) accuracy of the numerical method, and in Example 2 we consider a stationary state test for which we can verify the experimental order of convergence of the axisymmetric discrete formulation. Example 3 will present a pair of simulations of the full sedimentation-consolidation model in axisymmetric coordinates done under the assumptions of pointwise degeneracy of the diffusion coefficient as implied by (2.4) and under strong degeneracy (Examples 3a and 3b, respectively). In Example 4 we consider again pointwise degeneracy, axisymmetry, and the full sedimentationconsolidation model but replace the clarifier-thickener setup by a realistic secondary settling tank studied in literature.

4.1. Example 1: A model problem in Cartesian coordinates. For the first example, presented to evaluate the FVE formulation and to validate the implemented code, we define $\Omega:=(-1 / 2,1 / 2)^{2}$ and consider the flat two-dimensional problem of seeking $\boldsymbol{u}=\left(u_{1}, u_{2}\right)^{\mathrm{T}}, p$, and $\phi$ such that

$$
\begin{aligned}
\partial_{t} \phi+\nabla \cdot \boldsymbol{F}(\phi, \boldsymbol{u}) & =a \Delta \phi, \\
-\mu \Delta \boldsymbol{u}+\lambda \nabla p & =\boldsymbol{G}(\phi), \\
\lambda \nabla \cdot \boldsymbol{u} & =0,
\end{aligned}
$$

endowed with boundary conditions as follows: slip conditions $(\boldsymbol{u} \cdot \boldsymbol{n}=0)$ are applied for $\boldsymbol{u}$ on all parts of $\partial \Omega$, while for $\phi$ we prescribe zero-flux boundary conditions. The 

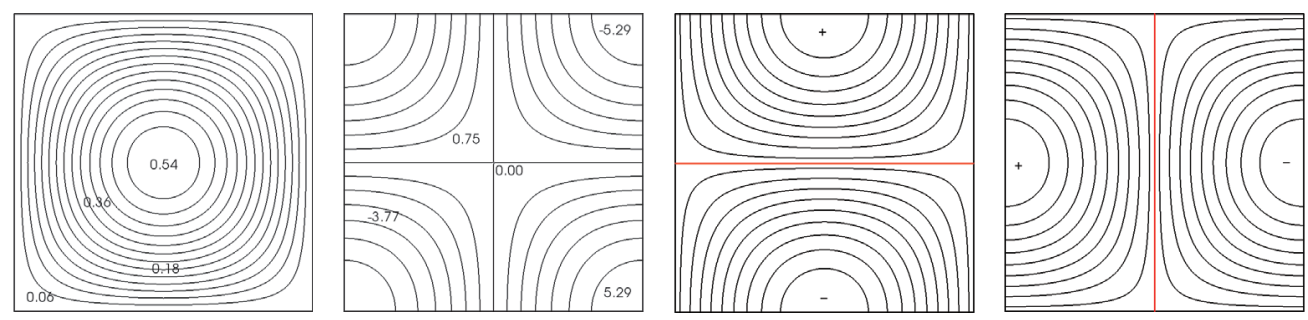

FIG. 3. Example 1: Approximate solutions for concentration, pressure field, and velocity components at time $t=1$ obtained with the FVE method. In this case, $u_{x, h}, u_{y, h} \in[-0.8409,0.8408]$, $\phi_{h} \in[0,0.5401]$, and $p_{h} \in[-5.28685,5.28685]$.

time interval is $t \in[0,1]$. The initial conditions are

$$
\boldsymbol{u}_{0}=\mathbf{0}, \quad \phi_{0}=-\cos (\pi x) \cos (\pi y),
$$

and the coefficients in (4.1) are chosen as

$$
\boldsymbol{G}=\left(0,-4 \pi^{2} \sin (\pi x) \cos (\pi y) \sin t\right)^{\mathrm{T}}, \quad \boldsymbol{F}(\phi, \boldsymbol{u})=\phi \boldsymbol{u}, \quad a=\frac{\tan t}{2 \pi^{2}}, \quad \mu=\lambda=1 .
$$

This choice leads to the vanishing of the convective term, i.e., $\nabla \cdot \boldsymbol{F}(\phi, \boldsymbol{u})=0$. The exact solution is

$$
\begin{aligned}
\phi(x, y, t) & =-\cos (\pi x) \cos (\pi y) \cos t, \quad p(x, y, t)=-2 \pi \sin (\pi x) \sin (\pi y) \sin t \\
u_{1}(x, y, t) & =\cos (\pi x) \sin (\pi y) \sin t, \quad u_{2}(x, y, t)=-\sin (\pi x) \cos (\pi y) \sin t .
\end{aligned}
$$

The errors to be studied are computed at the final time $T=1$ for $\boldsymbol{u}, p$, and $\phi$. The numerical solution is depicted in Figure 3. We focus on the accuracy in space only, so we apply the numerical method on several successively refined meshes. Let $\boldsymbol{v}_{h}$ and $q_{h}$ denote the FVE approximations of $\boldsymbol{v} \in \boldsymbol{H}^{1}(\Omega)$ and $q \in H^{1}(\Omega)$, respectively. We define relative errors by

$$
\begin{array}{ll}
e_{0}(\boldsymbol{v}):=\frac{\left\|\boldsymbol{v}-\boldsymbol{v}_{h}\right\|_{\boldsymbol{L}^{2}(\Omega)}}{\|\boldsymbol{v}\|_{L^{2}(\Omega)}}, & e_{1}(\boldsymbol{v}):=\frac{\left|\boldsymbol{v}-\boldsymbol{v}_{h}\right|_{\boldsymbol{H}^{1}(\Omega)}}{|\boldsymbol{v}|_{\boldsymbol{H}^{1}(\Omega)}}, \\
e_{0}(q):=\frac{\left\|q-q_{h}\right\|_{L^{2}(\Omega)}}{\|q\|_{L^{2}(\Omega)}}, & e_{1}(q):=\frac{\left|q-q_{h}\right|_{H^{1}(\Omega)}}{|q|_{H^{1}(\Omega)}}
\end{array}
$$

and let $r_{k}(\boldsymbol{v})$ and $r_{k}(q)$ denote the experimental rates of convergence given by

$$
r_{k}(\boldsymbol{v})=\frac{\log \left(e_{k}(\boldsymbol{v}) / \hat{e}_{k}(\boldsymbol{v})\right)}{\log (h / \hat{h})}, \quad r_{k}(q)=\frac{\log \left(e_{k}(q) / \hat{e}_{k}(q)\right)}{\log (h / \hat{h})}, \quad k \in\{0,1\},
$$

where $e_{k}$ and $\hat{e}_{k}$ stand for the corresponding errors obtained for two consecutive meshes of sizes $h$ and $\hat{h}$. Figure 4 indicates that the method approximately attains an $\mathcal{O}\left(h^{3 / 2}\right)$ order of convergence for $\phi$ in $L^{2}$, whereas an $\mathcal{O}(h)$ order is achieved for $\boldsymbol{u}$ in the $H^{1}$ seminorm and for $p$ in $L^{2}$. In the context of Stokes problems, with the chosen FE spaces these rates for $\boldsymbol{u}$ and $p$ are optimal (see, e.g., [48]), and the convergence rate for $\phi$ is also in accordance with previous results for linear problems [8].

Obviously, the regularity of the exact solution, along with the simplification of the model problem (notice that considering the uncoupled transport equation, the flux function is linear) are able to provide these rates, which are not recovered by the FVE approximation of the original problem (2.1)-(2.3). 


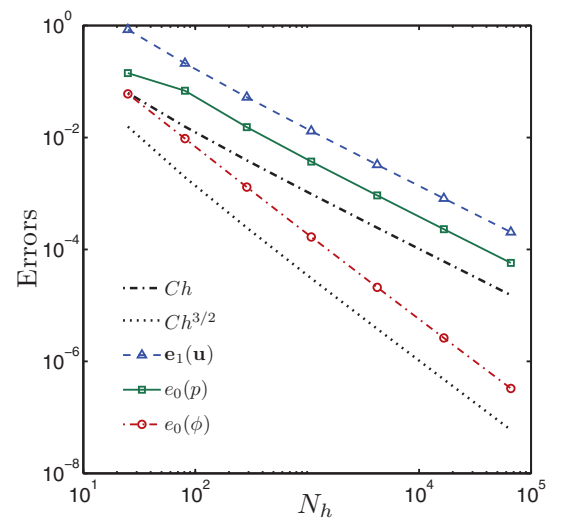

FIG. 4. Example 1: Convergence histories for the FVE method applied to the reduced model problem. The displayed quantities correspond to relative errors as defined in (4.2).

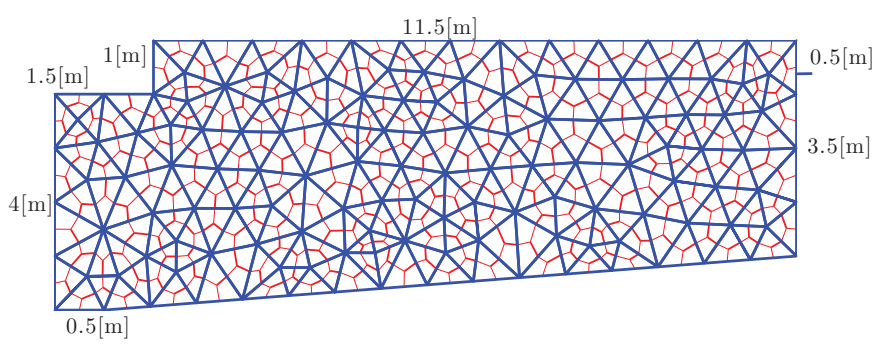

FIG. 5. Examples 2 and 3: Sketch of coarse primal and dual meshes for a clarifier unit. The inflow, outflow, and overflow boundaries $\Gamma_{\mathrm{in}}, \Gamma_{\mathrm{out}}, \Gamma_{\mathrm{c}}$ have lengths of $1.5,0.5$, and 0.5 meters, respectively. All other dimensions are also depicted.

4.2. Example 2: A steady-state problem. As a second example, and to assess the correctness of the axisymmetric scheme, we compute the steady-state solution the Stokes problem (2.2), (2.3) in the domain of interest. A coarse mesh $\mathcal{T}_{h}$ and the corresponding dual mesh $\mathcal{T}_{h}^{\star}$ are indicated in Figure 5. (The diamond mesh $\mathcal{T}_{h}^{\prime}$ is not drawn since it is not needed in the actual computations.) The dimensions of the clarifier-thickener are in the usual range for center-pie-like settling tanks.

Zero radial velocity is imposed on $\Gamma_{\mathrm{s}}$, and a parabolic profile is used as a boundary condition for $\boldsymbol{u}$ on $\Gamma_{\mathrm{in}}$. On $\Gamma_{\mathrm{c}}$ and $\Gamma_{\mathrm{out}}$ we apply parabolic profiles proportional to the size of the boundaries corresponding to the cylindrical unit, and on the remainder of the boundary we impose no-slip conditions.

In Figure 6 we present the numerical solution of the Stokes flow. To obtain approximate convergence rates for the numerical scheme, we compute approximate errors by using as a reference solution (replacing the unavailable exact solution) the numerical approximation obtained on highly refined primal and dual meshes (93,223 vertices and 184,972 elements in $\mathcal{T}_{h}$ ). These errors are computed in the norms associated to the spaces $\mathcal{V}$ and $Q$, that is,

$$
e_{1}^{a}(\boldsymbol{v}):=\frac{\left|\boldsymbol{v}-\boldsymbol{v}_{h}\right|_{\boldsymbol{H}_{1, \Gamma}^{1}(\Omega)}}{|\boldsymbol{v}|_{\boldsymbol{H}_{1, \Gamma}^{1}(\Omega)}}, \quad e_{0}^{a}(q):=\frac{\left\|q-q_{h}\right\|_{L_{1}^{2}(\Omega)}}{\|q\|_{L_{1}^{2}(\Omega)}},
$$

and $r_{1}^{a}(\boldsymbol{u}), r_{0}^{a}(p)$ denote the corresponding convergence rates. According to Table 1 , rates of convergence close to first order are obtained for $\boldsymbol{u}$ in the $\mathcal{V}$-norm and for $p$ 

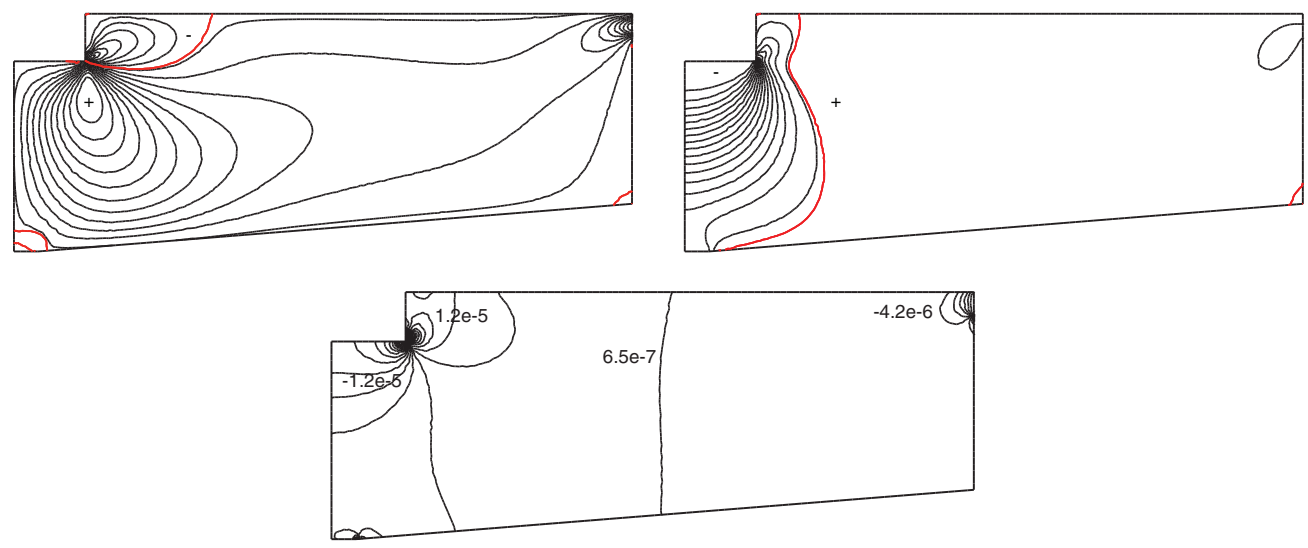

FIG. 6. Example 2: Steady-state solution of problem (2.2)-(2.3). Velocity (top) and pressure field (bottom). In this case, $u_{r, h} \in\left[-4.86 \times 10^{-5}, 5.70 \times 10^{-5}\right], u_{z, h} \in\left[-1.92 \times 10^{-4}, 2.11 \times 10^{-5}\right]$, and $p_{h} \in\left[-2.04 \times 10^{-5}, 2.06 \times 10^{-5}\right]$.

TABLE 1

Example 2: Number of interior nodes in the primal mesh $N_{h}$, approximate errors computed with respect to an FVE solution on a fine mesh, and experimental convergence rates for the steady state problem (2.2)-(2.3).

\begin{tabular}{ccccc}
\hline$N_{h}$ & $e_{1}^{a}(\boldsymbol{u})$ & $r_{1}^{a}(\boldsymbol{u})$ & $e_{0}^{a}(p)$ & $r_{0}^{a}(p)$ \\
\hline \hline 113 & $2.3016 \times 10^{-2}$ & - & $6.3122 \times 10^{-3}$ & - \\
403 & $1.0348 \times 10^{-2}$ & 0.9940 & $2.8831 \times 10^{-3}$ & 0.9972 \\
1508 & $4.7215 \times 10^{-3}$ & 0.9762 & $1.2914 \times 10^{-3}$ & 1.0239 \\
6030 & $2.0368 \times 10^{-3}$ & 0.9947 & $5.5939 \times 10^{-4}$ & 0.9673 \\
22931 & $8.9410 \times 10^{-4}$ & 0.9968 & $2.3896 \times 10^{-4}$ & 0.9816 \\
\hline
\end{tabular}

in the $Q$-norm. This behavior is expected for $\mathrm{FE}$ approximations of axisymmetric Stokes equations (see [3, Theorem 5]), although the result in [3] (based on some of the regularity assumptions of $[6$, section IX.1]) is valid for convex domains only.

4.3. Example 3: A clarifier-thickener simulation. We now present the numerical solution of (2.1)-(2.3) for the clarifier-thickener unit. From now on, all component velocities are given in $[\mathrm{m} / \mathrm{s}]$ and pressure in $[\mathrm{Pa}]$. The model and numerical parameters are displayed in Table 2. In principle, the stabilization parameters $\kappa_{F}^{\phi}$, $\left(\kappa_{K}^{p}\right)^{-1}$, and $\left(\kappa_{F}^{\boldsymbol{u}}\right)^{-1}$ are unfortunately problem dependent and should be tuned so that they are as small as possible while maintaining stability.

Example 3a. The initial datum for $\phi$ is $\phi=0$ in the whole vessel. The boundary conditions are set as follows. The volumetric bulk flow rate through the generic part of the boundary $\tilde{\Gamma}_{i}$ (of the three-dimensional domain) is given by $Q_{i}=\int_{\tilde{\Gamma}_{i}} \tilde{\boldsymbol{u}} \cdot \boldsymbol{n} \mathrm{d} \sigma$, where in cylindrical coordinates $\tilde{u}_{r}=u_{r}, \tilde{u}_{z}=u_{z}$ and $\tilde{u}_{\theta}=0$ (see section 2.2). At the inlet $\Gamma_{\text {in }}$ we set $\boldsymbol{u}_{\text {in }}=\left(0,-u_{z, \text { in }}\right)^{\mathrm{T}}$, meaning that the vessel is continuously fed with feed suspension of concentration $\phi_{\text {in }}$. This gives $Q_{\text {in }}=-u_{z, \text { in }}\left|\tilde{\Gamma}_{\text {in }}\right|$, where $\left|\tilde{\Gamma}_{\text {in }}\right|=\pi\left|\Gamma_{\text {in }}\right|^{2}=\frac{9 \pi}{4}\left[\mathrm{~m}^{2}\right]$ is the area of the inlet boundary $\tilde{\Gamma}_{\text {in }}$. At the outlet $\Gamma_{\text {out }}$ we prescribe $\boldsymbol{u}_{\text {in }}=\left(0,-u_{z \text {, out }}\right)^{\mathrm{T}}$, which indicates that the concentrated sediment is removed from the unit at a volume underflow rate

$$
Q_{\text {out }}=u_{z, \text { out }}\left|\tilde{\Gamma}_{\text {out }}\right| \text { with }\left|\tilde{\Gamma}_{\text {out }}\right|=\pi\left|\Gamma_{\text {out }}\right|^{2}=\frac{\pi}{4}\left[\mathrm{~m}^{2}\right] .
$$


TABLE 2

Examples 3 and 4: List of geometrical data, and model and numerical parameters considered in the simulations.

\begin{tabular}{|c|c|}
\hline Quantity & Values \\
\hline Density difference & $\Delta \varrho=1562\left[\mathrm{~kg} / \mathrm{m}^{3}\right]$ \\
\hline \multicolumn{2}{|l|}{ Size of the unit } \\
\hline Example 3 & Sectional area $=70.5\left[\mathrm{~m}^{2}\right]($ Figure 5$)$ \\
\hline Example 4 & Sectional area $=53.3\left[\mathrm{~m}^{2}\right]$ \\
\hline Maximum volume fraction and gel point & $\phi_{\max }=0.9, \phi_{\mathrm{c}}=0.1$ \\
\hline Settling velocity in an unbounded medium & $u_{\infty}=2.2 \times 10^{-3}[\mathrm{~m} / \mathrm{s}]$ \\
\hline Gravity force & $g=9.81\left[\mathrm{~m} / \mathrm{s}^{2}\right]$ \\
\hline Prescribed concentration at inlet & $\phi_{\text {in }}=0.08$ \\
\hline Absolute bulk boundary velocities & $u_{z, \mathrm{out}}=\nu u_{z, \mathrm{in}}, u_{r, \mathrm{ofl}}=\frac{9-\nu}{52} u_{z, \text { in }}$ \\
\hline Example 3 & $u_{z, \text { in }}=2.9 \times 10^{-3}[\mathrm{~m} / \mathrm{s}], \nu=0$ \\
\hline Example 4 & $u_{r, \text { in }}=1.9 \times 10^{-2}[\mathrm{~m} / \mathrm{s}], \nu=0.75$ \\
\hline Other model parameters & $\alpha=5, \beta=2.5, \lambda=1, \sigma_{0}=5 \times 10^{-2}$ \\
\hline \multicolumn{2}{|l|}{ Meshsize and time step } \\
\hline Example 3 & $h=0.1428[\mathrm{~m}], \Delta t=1.0[\mathrm{~s}]$ \\
\hline Example 4 & $h=0.0961[\mathrm{~m}], \Delta t=5.0[\mathrm{~s}]$ \\
\hline Stabilization parameters & $\kappa_{F}^{\phi}=\frac{1}{12}, \kappa_{F}^{u}=100$ \\
\hline Example 3 & $\kappa_{K}^{p}=200$ \\
\hline Example 4 & $\kappa_{K}^{p}=500$ \\
\hline
\end{tabular}

On the symmetry axis $\Gamma_{\mathrm{s}}$ we put $u_{r}=0$. Consistently with the global conservation of mass of the mixture we set

$$
Q_{\text {ofl }}=Q_{\text {out }}-Q_{\text {in }}=u_{z, \text { out }} \frac{\pi}{4}+u_{z, \text { in }} \frac{9 \pi}{4},
$$

and then, from $Q_{\text {ofl }}=u_{r, \text { of }}\left|\tilde{\Gamma}_{\mathrm{c}}\right|$ and $\left|\tilde{\Gamma}_{\mathrm{c}}\right|=2 \pi R\left|\Gamma_{\mathrm{c}}\right|=13 \pi\left[\mathrm{m}^{2}\right]$, at the overflow we impose $\boldsymbol{u}_{\mathrm{ofl}}=\left(u_{r, \text { ofl }}, 0\right)^{\mathrm{T}}$, where

$$
u_{r, \text { of }}=\frac{u_{z, \text { out }}\left|\tilde{\Gamma}_{\text {out }}\right|}{\left|\tilde{\Gamma}_{\mathrm{c}}\right|}+\frac{u_{z, \text { in }}\left|\tilde{\Gamma}_{\text {in }}\right|}{\left|\tilde{\Gamma}_{\mathrm{c}}\right|}=\frac{9}{52} u_{z, \text { out }}+\frac{1}{52} u_{z, \text { in }} .
$$

On the remainder of $\partial \Omega$ we enforce a no-slip condition (homogeneous Dirichlet boundary data for $\boldsymbol{u}$ ) and zero-flux boundary conditions for $\phi$. The primal mesh $\mathcal{T}_{h}$ used in the experiments consists of 8708 elements and 4516 interior nodes. As the time step we use $\Delta t=1.0[\mathrm{~s}]$, which is sufficiently accurate to capture the main phenomena of the problem.

Figure 7 shows the volume fraction profiles and Figure 8 shows the corresponding velocity fields at different times. After $t=1000[\mathrm{~s}]$, the effect of sedimentation is clearly noticed since $\phi$ increases toward the bottom of the tank. The concentration at the effluent remains equals to zero until $t=10[\mathrm{~h}]$.

Example 3b. In light of Remark 2.1 we perform an analogous simulation with a diffusion function $A$ that vanishes for $\phi \leq \phi_{\mathrm{c}}$. Condition (2.4) is replaced by the choice

$$
\sigma_{\mathrm{e}}^{\prime}(\phi)=\left\{\begin{array}{ll}
0 & \text { for } \phi \leq \phi_{\mathrm{c}}, \\
\sigma_{0} \frac{\alpha}{\phi_{\mathrm{c}}}\left(\frac{\phi}{\phi_{\mathrm{c}}}\right)^{\alpha-1} & \text { otherwise, }
\end{array} \quad \sigma_{0}, \alpha>0, \quad 0<\phi_{\mathrm{c}} \leq 1 .\right.
$$

Figure 9 presents the concentration profiles and Figure 10 presents the corresponding velocity fields at four different times. The sedimentation front is now sharper than 

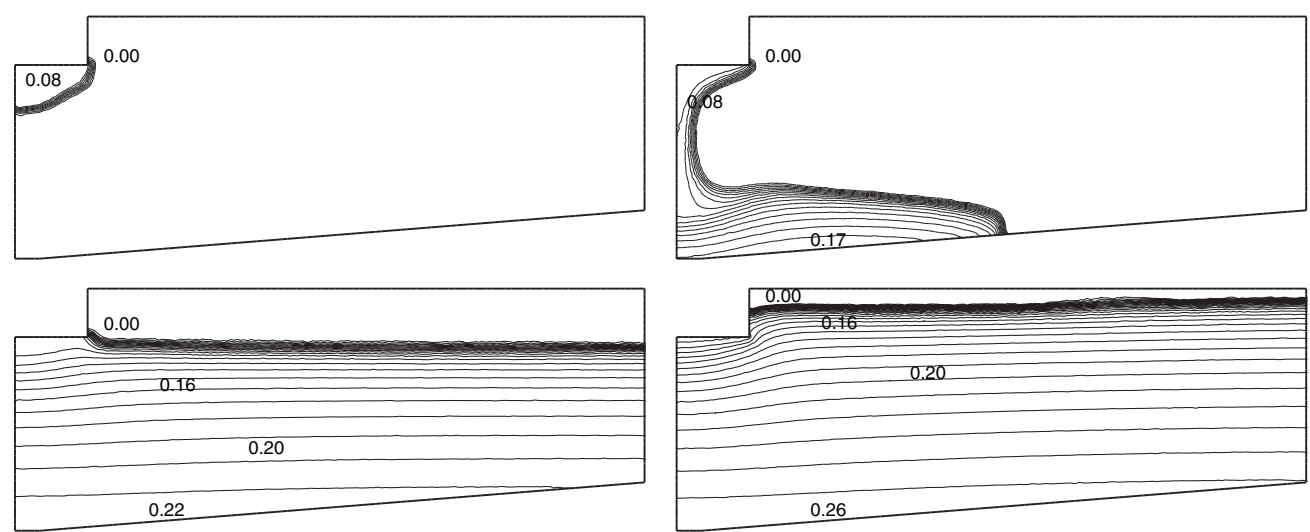

FIG. 7. Example 3a: Snapshots of the numerical solution for the clarifier-thickener problem: concentration profiles at times $t=100[\mathrm{~s}]$ (top left), $t=1000[\mathrm{~s}]$ (top right), $t=8000[\mathrm{~s}]$ (bottom left), and $t=30,000[\mathrm{~s}]$ (bottom right). The contours correspond to $\phi=0.01$, $0.02, \ldots, 0.09,0.1,0.12,0.14, \ldots, 0.26$.
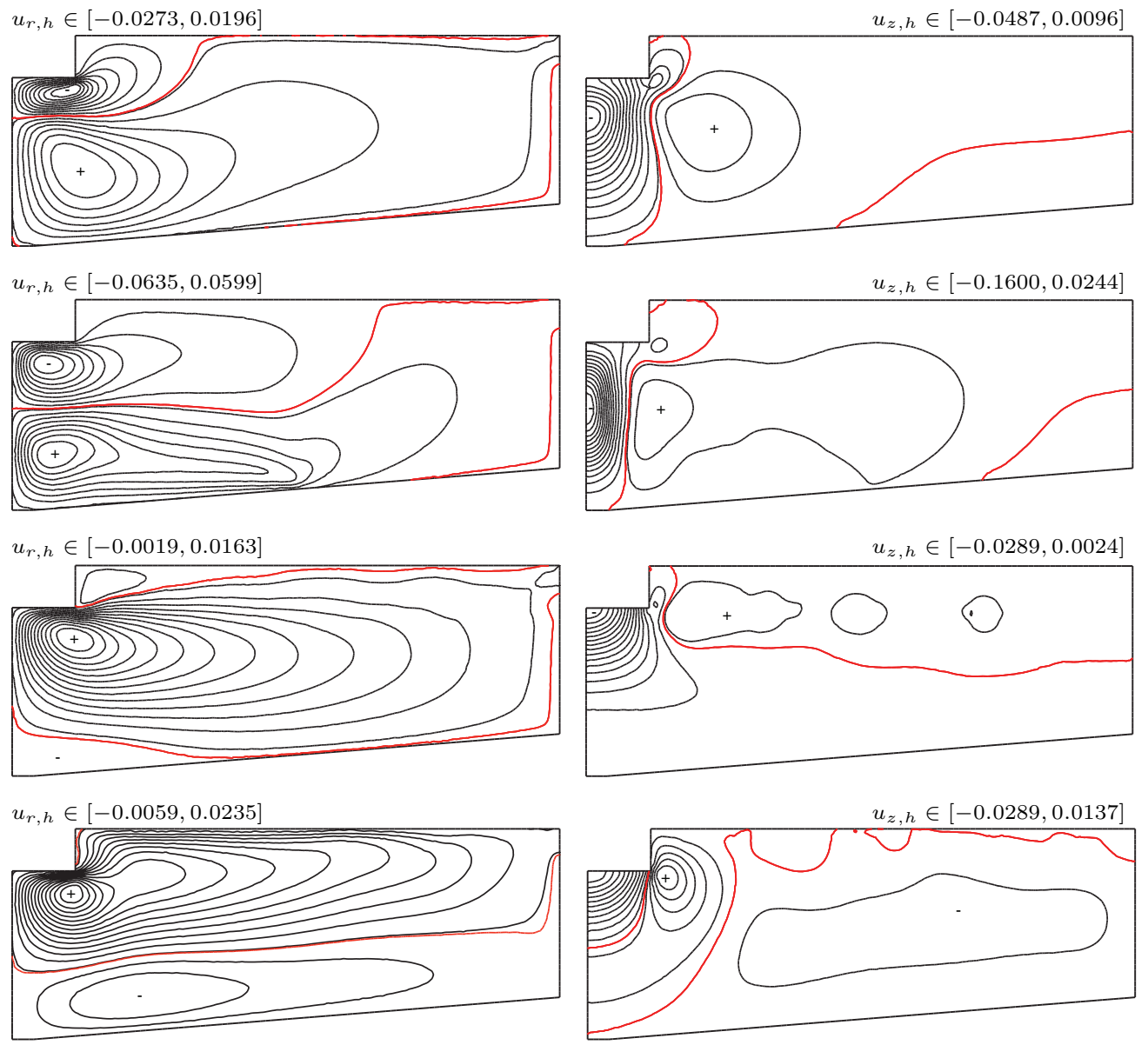

Fig. 8. Example 3a: Snapshots of the numerical solution for the clarifier-thickener problem: velocity components (radial and height, in left and right columns, respectively) at times $t=100[\mathrm{~s}]$, $t=1000[\mathrm{~s}], t=8000[\mathrm{~s}]$, and $t=30,000[\mathrm{~s}]$. 

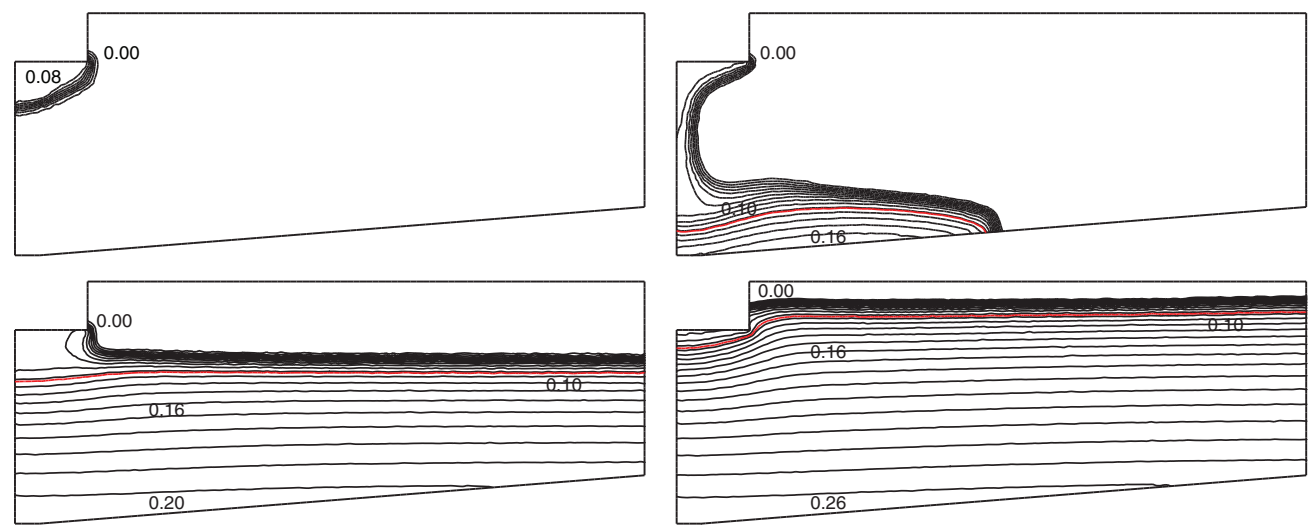

FIG. 9. Example 3b: Snapshots of the numerical solution for the clarifier-thickener problem with degenerate diffusion. Concentration profiles at times $t=100[\mathrm{~s}]$ (top left), $t=1000[\mathrm{~s}]$ (top right), $t=8000[\mathrm{~s}]$ (bottom left), and $t=30,000[\mathrm{~s}]$ (bottom right). The contours correspond to $\phi=0.01,0.02, \ldots, 0.09,0.1,0.12,0.14, \ldots, 0.26$.
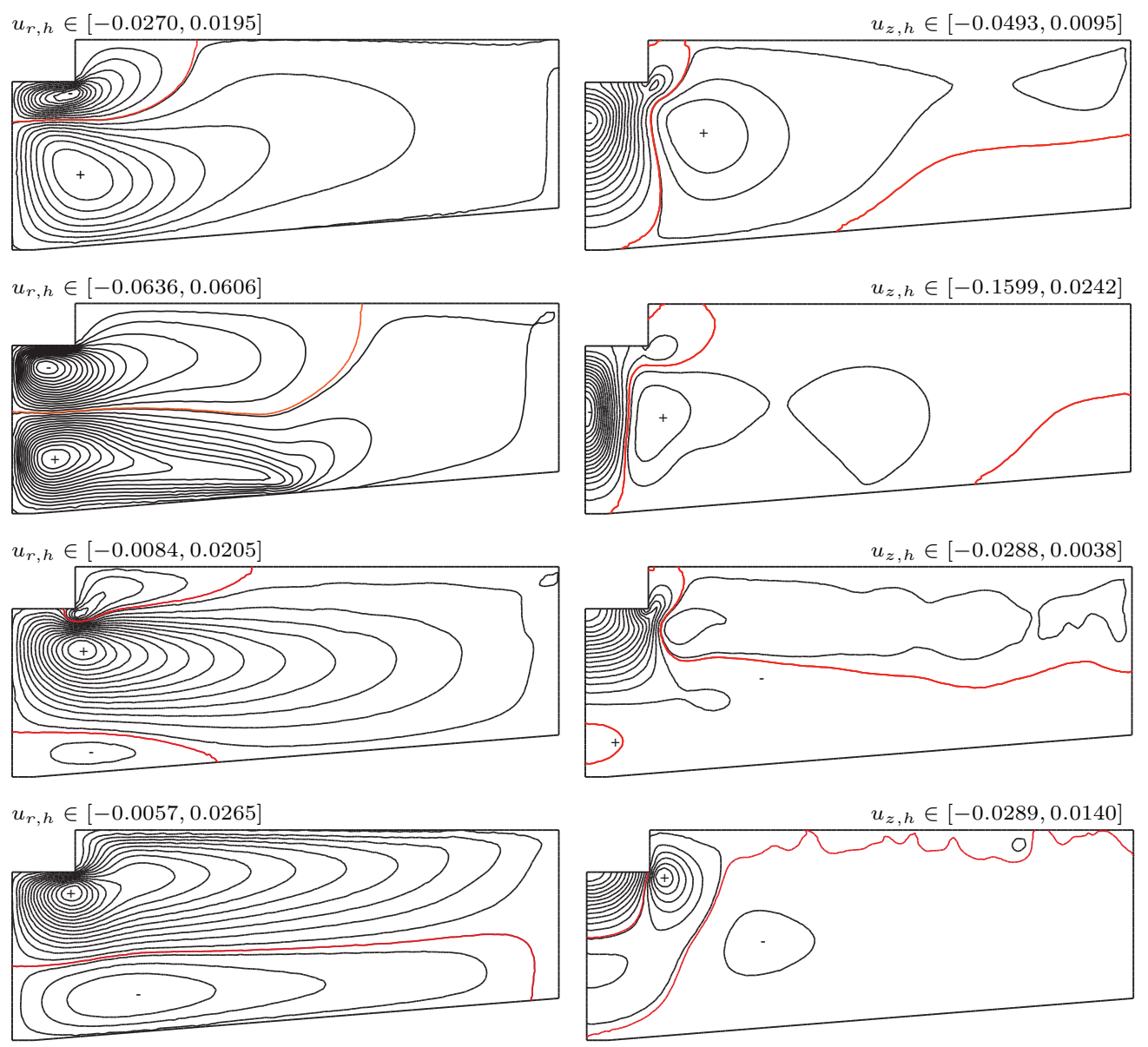

FIG. 10. Example 3b: Snapshot of the numerical solution for the clarifier-thickener problem with degenerate diffusion: velocities at times $t=100[\mathrm{~s}], t=1000[\mathrm{~s}], t=8000[\mathrm{~s}]$, and $t=30,000[\mathrm{~s}]$ (from top to bottom). 


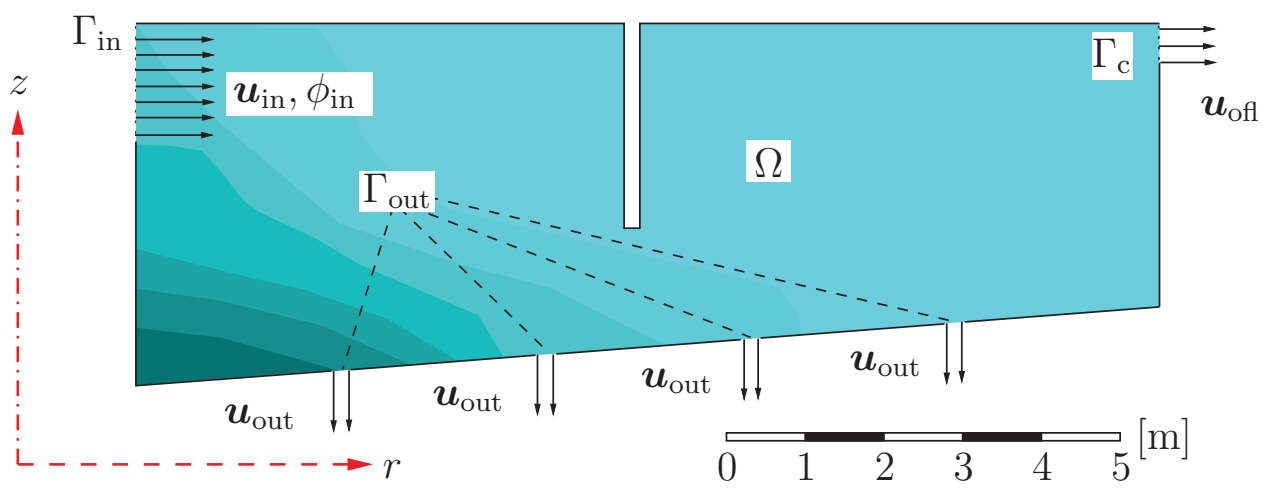

FIG. 11. Example 4: secondary settling tank studied in $[37,55]$. The device has a center feed, peripheral and radial effluent overflows, and a skirt baffle acting as flocculator. For this example, in the bottom of the tank there are four suction lifts for the sludge of width $0.25[\mathrm{~m}]$ each. The unit has a radial length and height of $13[\mathrm{~m}]$ and $4.6[\mathrm{~m}]$, respectively, the inlet has a height of $1.50[\mathrm{~m}]$, the baffle has a width of $0.2[\mathrm{~m}]$ and a height of $2.6[\mathrm{~m}]$, the effluent outlet has a height of $0.5[\mathrm{~m}]$, and the slope of the bottom of the tank is of $6.5 \%$.

in the previous example (Example 3a), and the FVE method is still able to resolve it accurately, without changing the discretization parameters.

4.4. Example 4: A secondary settling unit. We consider a secondary settling tank studied in $[37,55]$. (See Figure 11, where the geometry is also described.) The difference to the clarifier-thickener configuration is that the feed inlet is oriented radially rather than axially; the outlet is associated with four so-called suction lifts rather than a central discharge; and there is a so-called skirt baffle separating the area closer to the inlet from that farther away.

For our simulation all values for the parameters are considered as in Table 2 . The primal mesh $\mathcal{T}_{h}$ is composed by 7410 elements and 4206 interior nodes. The boundary conditions for velocity at the suction lifts are given by $\boldsymbol{u}=\left(0,-u_{z, \text { out }} / 4\right)$, where $u_{z, \text { out }}=\nu u_{r, \text { in }}$ with $\nu=0.75$.

The evolution of the concentration fields is depicted in Figure 12, where we present profiles corresponding to time instants $t=1000,3000,7500,15,000[\mathrm{~s}]$. We observe from the corresponding velocity profiles, shown in Figure 13, that from time $t=$ $7500[\mathrm{~s}]$ the material starts to be extracted at the bottom lifts, which explains the decrease in the concentration in the bottom.

5. Concluding remarks. In the present work, an innovative numerical technique for the solution of a coupled fluid flow and transport problem in a two-dimensional axisymmetric domain has been proposed within the context of sedimentationconsolidation models. It is of practical importance to solve accurately the reduced two-dimensional problem to model the underlying full three-dimensional phenomenon.

The numerical scheme introduced is based on a low-order stabilized FVE discretization, where a novel ingredient is the use of two different dual meshes for the velocity and the concentration field. We have verified the convergence of the proposed numerical method in simple test cases and have performed relevant transient simulations on the axisymmetric geometry. Similar problems might be found in several other application fields, and therefore this technique can be, and is currently being, extended to accommodate the study of related and more involved models. We mention that without much effort, a step forward could be made in including, for 

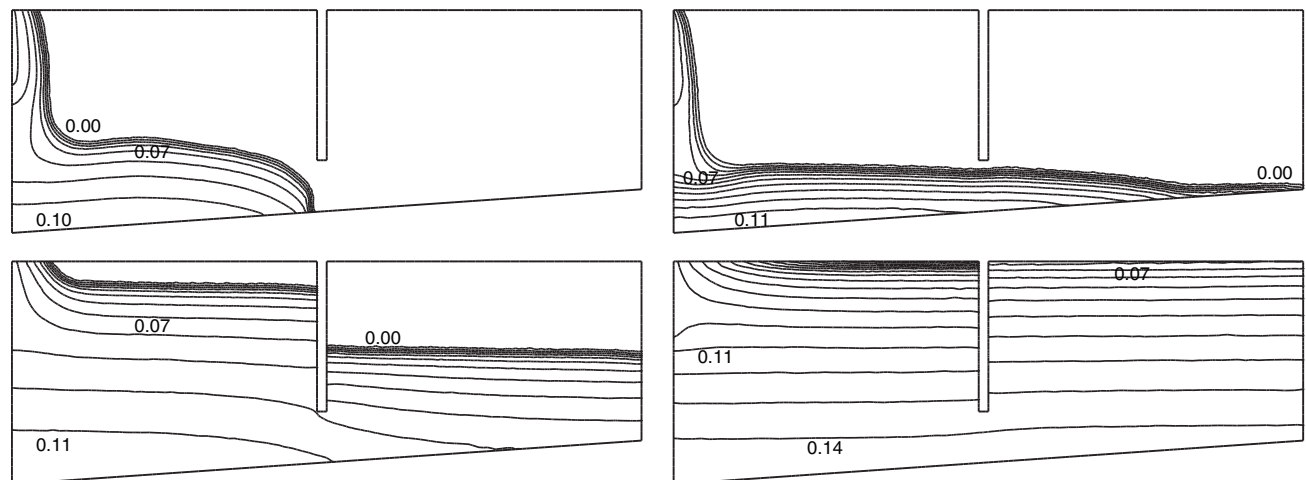

FIG. 12. Example 4: Snapshot of the numerical solution for the clarifier-thickener problem: concentration fields at times $t=1000[\mathrm{~s}]$ (top left), $t=3000[\mathrm{~s}]$ (top right), $t=7500[\mathrm{~s}]$ (bottom left), and $t=15,000[\mathrm{~s}]$ (bottom right). The contours correspond to $\phi=0.01,0.02, \ldots, 0.12,0.13,0.14$.
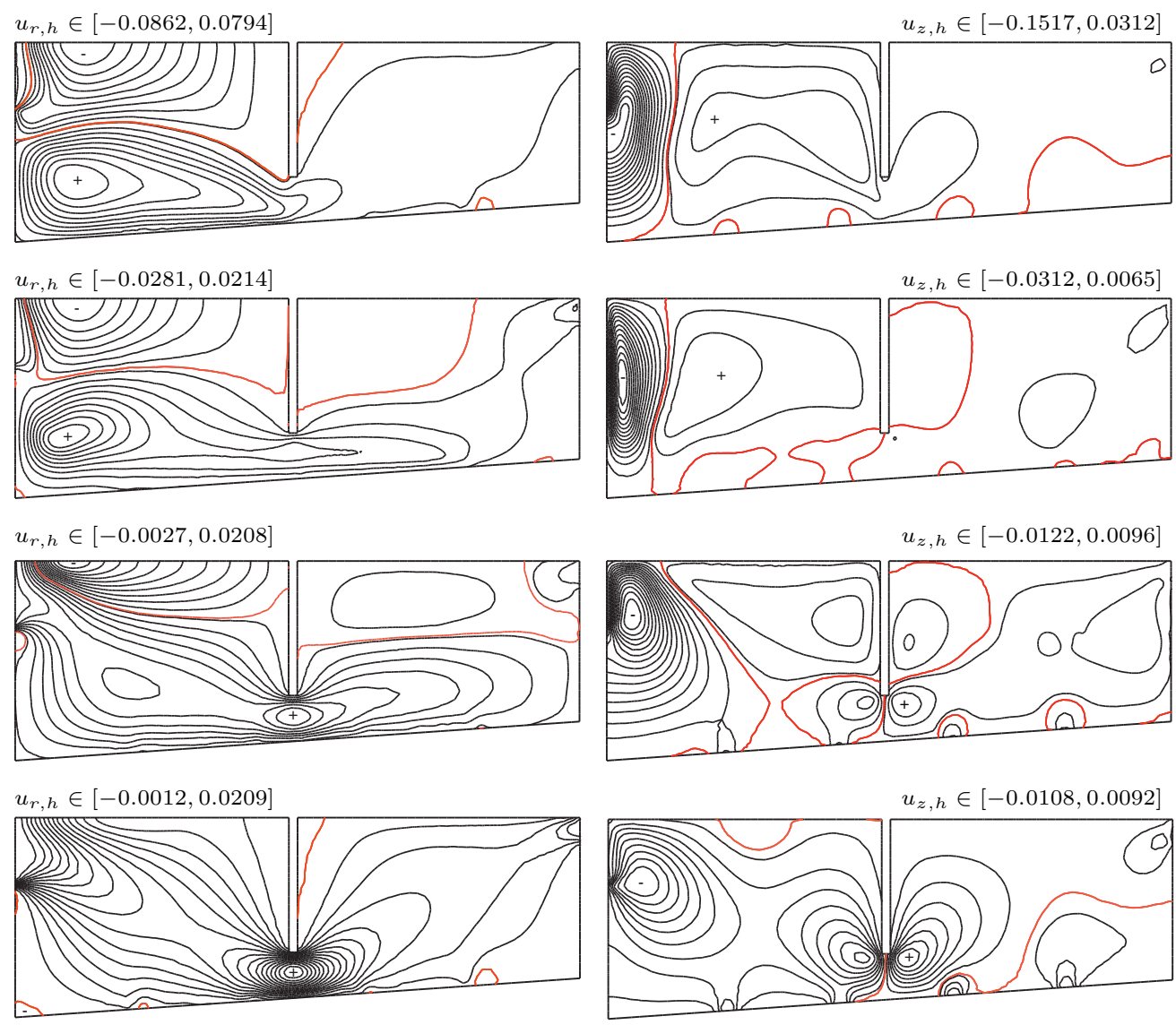

FIG. 13. Example 4: Snapshot of the numerical solution for the clarifier-thickener problem: velocity fields at times $t=1000[\mathrm{~s}], t=3000[\mathrm{~s}], t=7500[\mathrm{~s}]$, and $t=15,000[\mathrm{~s}]$ (from top to bottom). 
instance, more general rheological assumptions [40], and the sedimentation of polydisperse flocculated suspensions [9]. A further issue at the numerical level is the pointwise satisfaction of the divergence-free condition. In this regard, a possible improvement to our model could be the formulation of FVE discretizations employing features of the Scott-Vogelius mixed finite elements.

Acknowledgments. We thank two anonymous referees for their valuable comments, which resulted in a number of improvements of this paper.

\section{REFERENCES}

[1] B. Andreianov, F. Boyer, And F. Hubert, Discrete duality finite volume schemes for LerayLions-type elliptic problems on general $2 D$ meshes, Numer. Methods Partial Differential Equations, 23 (2007), pp. 145-195.

[2] R. Araya, G. Barrenechea, and F. Valentin, A stabilized finite-element method for the Stokes problem including element and edge residuals, IMA J. Numer. Anal., 27 (2007), pp. 172-197.

[3] Z. Belhachmi, C. Bernardi, and S. Deparis, Weighted Clément operator and application to the finite element discretization of the axisymmetric Stokes problem, Numer. Math., 105 (2006), pp. 217-247.

[4] Z. Belhachmi, C. Bernardi, S. Deparis, and F. Hecht, An efficient discretization of the Navier-Stokes equations in an axisymmetric domain. Part 1: The discrete problem and its numerical analysis, J. Sci. Comput., 27 (2006), pp. 97-110.

[5] F. Benkhaldoun, S. Sahmin, And M. Seaid, Solution of the sediment transport equations using a finite volume method based on sign matrix, SIAM J. Sci. Comput., 31 (2009), pp. 2866-2889.

[6] C. Bernardi, M. Dauge, and Y. Maday, Spectral Methods for Axisymmetric Domains, Gauthier-Villars, Paris, 1999.

[7] C. Bi And M. Liu, A discontinuous finite volume element method for second-order elliptic problems, Numer. Methods Partial Differential Equations, 28 (2012), pp. 425-440.

[8] F. BrezZi, L.D. Marini, ANd E. SüLI, Discontinuous Galerkin methods for first-order hyperbolic problems, Math. Models Methods Appl. Sci., 14 (2004), pp. 1893-1903.

[9] S. Berres, R. Bürger, K.H. Karlsen, and E.M. Tory, Strongly degenerate parabolichyperbolic systems modeling polydisperse sedimentation with compression, SIAM J. Appl. Math., 64 (2003), pp. 41-80.

[10] R. Bürger, K.H. KARlSen, H. Torres, And J.D. Towers, Second order schemes for conservation laws with discontinuous flux modelling clarifier-thickener units, Numer. Math., 116 (2010), pp. 579-617.

[11] R. Bürger, R. Ruiz-Baier, K. Schneider, and H. Torres, Adaptive multiresolution schemes for two-dimensional sedimentation processes, Int. J. Numer. Anal. Model., 9 (2012), pp. $479-504$.

[12] R. Bürger, W.L. Wendland, And F. Concha, Model equations for gravitational sedimentation-consolidation processes, ZAMM Z. Angew. Math. Mech., 80 (2000), pp. 79-92.

[13] E. Burman, A. Quarteroni, ANd B. Stamm, Interior penalty continuous and discontinuous finite element approximations of hyperbolic equations, J. Sci. Comput., 43 (2010), pp. 293312.

[14] M.C. Bustos, F. Concha, R. Bürger, and E.M. Tory, Sedimentation and Thickening, Kluwer Academic, Dordrecht, The Netherlands, 1999.

[15] Z. CAI, On the finite volume element method, Numer. Math., 58 (1991), pp. 713-735.

[16] C. Calgaro, E. Creusé, and T. Goudon, An hybrid finite volume-finite element method for variable density incompressible flows, J. Comput. Phys., 227 (2008), pp. 4671-4696.

[17] J.H. Carneiro de Araujo and V. Ruas, A stable finite element method for the axisymmetric three-field Stokes system, Comput. Methods Appl. Mech. Engrg., 164 (1998), pp. 267-286.

[18] A. ÇeşmelioĞLu And B. Rivière, Primal discontinuous Galerkin methods for time-dependent coupled surface and subsurface flow, J. Sci. Comput., 40 (2009), pp. 115-140.

[19] P. Chatzipantelidis, V. Ginting, and R.D. Lazarov, A finite volume element method for a non-linear elliptic problem, Numer. Linear Algebra Appl., 12 (2005), pp. 515-546.

[20] C. Choquet and S. Zimmermann, Analysis of a finite-volume-finite-element scheme for a nuclear transport model, IMA J. Numer. Anal., 31 (2011), pp. 86-115. 
[21] M.A. Christon, P.M. Gresho, And S.B. Sutton, Computational predictability of timedependent natural convection flows in enclosures (including a benchmark solution), Internat. J. Numer. Methods Fluids, 40 (2002), pp. 953-980.

[22] B. Cockburn And C.-W. Shu, Runge-Kutta discontinuous Galerkin methods for convectiondominated problems, J. Sci. Comput., 16 (2001), pp. 173-261.

[23] T.A. DAVIS, An unsymmetric-pattern multifrontal method for sparse $L U$ factorization, SIAM J. Matrix Anal. Appl., 18 (1997), pp. 140-158.

[24] A. Deininger, E. Holthausen, and P.A. Wilderer, Velocity and solids distribution in circular secondary clarifiers: Full scale measurements and numerical modelling, Water Res., 32 (1998), 2951-2958.

[25] D.A. Di Pietro And A. ERn, Mathematical Aspects of Discontinuous Galerkin Methods, Springer-Verlag, Berlin, 2012.

[26] S. DiEHL, Dynamic and steady-state behaviour of continuous sedimentation, SIAM J. Appl. Math., 57 (1997), pp. 991-1018.

[27] S. DieHL, Operating charts for continuous sedimentation IV: Limitations for control of dynamic behaviour, J. Engrg. Math., 60 (2008), pp. 249-264.

[28] S. DieHL, The solids-flux theory - confirmation and extension by using partial differential equations, Water Res., 42 (2008), pp. 4976-4988.

[29] G.A. Ekama, J.L. Barnard, F.W. Günthert, P. Krebs, J.A. McCorquodale, D.S. Parker, And E.J. WAhlberg, Secondary Settling Tanks: Theory, Modelling, Design and Operation. Scientific and Technical report 6, International Association on Water Quality, London, 1997.

[30] G.A. Ekama And P. Marais, Assessing the applicability of the $1 D$ flux theory to full-scale secondary settling tank design with a 2 D hydrodynamic model, Water Res., 38 (2004), pp. 495-506.

[31] M. Flǘk, T. Hofer, M. Picasso, J. Rappaz, and G. Steiner, Scientific computing for aluminum production, Int. J. Numer. Anal. Model., 6 (2009), pp. 489-504.

[32] L.P. Franca, S.L. Frey, and T. Hughes, Stabilized finite element methods. I: Application to advective-diffusive problems, Comput. Methods Appl. Mech. Engrg., 95 (1992), pp. 253271.

[33] L. Gastaldo, R. Herbin, and J.-C. Latché, An unconditionally stable finite element-finite volume pressure correction scheme for the drift-flux model, M2AN Math. Model. Numer. Anal. 44 (2010), pp. 251-287.

[34] E.H. Georgoulis, E. Hall, and J.M. Melenk, On the suboptimality of the p-version interior penalty discontinuous Galerkin method, J. Sci. Comput., 42 (2010), pp. 54-67.

[35] A. Guardone and L. Vigevano, Finite element/volume solution to axisymmetric conservation laws, J. Comput. Phys., 224 (2007), pp. 489-518.

[36] G. Jouvet, M. Huss, M. Picasso, J. Rappaz, and H. Blatter, Numerical simulation of Rhone's glacier from 1874 to 2100, J. Comput. Phys., 228 (2009), pp. 6426-6439.

[37] D. Kleine And B.D. RedDy, Finite element analysis of flows in secondary settling tanks, Internat. J. Numer. Methods Engrg., 64 (2005), pp. 849-876.

[38] G.J. KYNCH, A theory of sedimentation, Trans. Faraday Soc., 48 (1952), pp. 166-176.

[39] O.A. Ladyzhenskaya, V. Solonnikov, and N. Ural' ceva, Linear and Quasi-Linear Equations of Parabolic Type, American Mathematical Society, Providence, RI, 1968.

[40] D.R. Lester, M. Rudman, and P.J. Scales, Macroscopic dynamics of flocculated colloidal suspensions, Chem. Engrg. Sci., 65 (2010), pp. 6362-6378.

[41] G. Mazzolani, F. Pirozzi, And G. D'AntonoI, A generalized settling approach in the numerical modeling of sedimentation tanks, Water Sci. Tech., 38 (1998), pp. 95-102.

[42] B. Mercier AND G. Raugel, Resolution d'un problème aux limites dans un ouvert axisymétrique par éléments finis en $r, z$ et séries de Fourier en $\theta$, RAIRO Anal. Numér., 16 (1982), pp. 405-461.

[43] S. McGee and P. Seshaiyer, Finite difference methods for coupled flow interaction transport models, Electron. J. Differ. Equ. Conf., 17 (2009), pp. 171-184.

[44] D.S. McLachlan, Equation for the conductivity of microscopic mixtures, J. Phys. C Solid State Phys., 19 (1986), pp. 1339-1345.

[45] A.S. Michaels And J.C. Bolger, Settling rates and sediment volumes of flocculated Kaolin suspensions, Ind. Engrg. Chem. Fund., 1 (1962), pp. 24-33.

[46] M.S. Nigam, Numerical simulation of buoyant mixture flows, Int. J. Mult. Flow, 29 (2003), pp. 983-1015.

[47] K.V. PARchevsky, Numerical simulation of sedimentation in the presence of $2 D$ compressible convection and reconstruction of the particle-radius distribution function, J. Engrg. Math., 41 (2001), pp. 203-219. 
[48] A. Quarteroni And R. Ruiz-Baier, Analysis of a finite volume element method for the Stokes problem, Numer. Math., 118 (2011), pp. 737-764.

[49] R.R. RaO, L.A. Mondy, And S.A. Altobelli, Instabilities during batch sedimentation in geometries containing obstacles: A numerical and experimental study, Internat. J. Numer. Methods Fluids, 55 (2007), pp. 723-735.

[50] J.N. Reddy And D.K. GartLing, The Finite Element Method in Heat Transfer and Fluid Dynamics, CRC Press, Boca Raton, FL, 2001.

[51] L. Shen ANd Z.Chen, Analysis of a stabilized finite volume method for the transient Stokes equations, Int. J. Numer. Anal. Model., 6 (2009), pp. 505-519.

[52] M. TABATA AND A. Suzuki, A stabilized finite element method for the Rayleigh-Bénard equations with infinite Prandtl number in a spherical shell, Comput. Methods Appl. Mech. Engrg., 190 (2000), pp. 387-402.

[53] E.M. Tory, ED., Sedimentation of Small Particles in a Viscous Fluid, Computational Mechanics Publications, Southampton, UK, 1996.

[54] M. Ungarish, Hydrodynamics of Suspensions, Springer-Verlag, Berlin, 1993.

[55] R.W. Watts, S.A. Svoronos, and B. Koopman, One dimensional modelling of secondary clarifiers using a concentration feed velocity dependent dispersion coefficient, Water Res., 30 (1996), pp. 2112-2124. 